\title{
Lamiaceae de México
}

\author{
Martha Martínez-Gordillo1, Brenda Bedolla-García², Guadalupe Cornejo- \\ Tenorio ${ }^{3}$, Itzl Fragoso-Martínez ${ }^{4 *}$, María del Rosario García-Peña ${ }^{4}$, Jesús \\ Guadalupe González-Gallegos ${ }^{5}$, Sabina I. Lara-Cabrera ${ }^{6}$ y Sergio Zamudio ${ }^{7}$
}

Botanical Sciences 95 (4): 780-806, 2017

DOI: $10.17129 /$ botsci.1871

Received:

2 de octubre de 2017

Accepted:

10 de noviembre de 2017

Associate Editor: Guillermo Ibarra Manriquez

Copyright: (C) 2017 Martínez-Gordillo et al. This is an open access article distributed under the terms of the Creative Commons Attribution License, which permits unrestricted use, distribution, and reproduction in any medium, provided the original author and source are credited.

Herbario de la Facultad de Ciencias (FCME), Universidad Nacional Autónoma de México. Ciudad de México.

2 Instituto de Ecología A.C., Centro Regional del Bajío. Pátzcuaro, Michoacán.

3 Instituto de Investigaciones en Ecosistemas y Sustentabilidad, Universidad Nacional Autónoma de México. Morelia, Michoacán.

${ }^{4}$ Herbario Nacional de México. Instituto de Biología, Universidad

Nacional Autónoma de México. Ciudad de México.

${ }_{5}^{5}$ CONACYT — Instituto Politécnico Nacional, Centro Interdisciplinario de Investigación para el Desarrollo Integral Regional, CIIDIR - Durango, Victoria de Durango, Durango.

${ }^{6}$ Laboratorio de Sistemática Molecular, Facultad de Biología, Universidad Michoacana de San Nicolás de Hidalgo. Morelia, Michoacán.

${ }^{7}$ Apartado Postal 392, Pátzcuaro 61600, Michoacán, México.

*Autor de correspondencia:

i.fragoso@ciencias.unam.mx

\section{Resumen}

Antecedentes: Lamiaceae es una de las familias con mayor riqueza de especies en México. Sin embargo, aún se carece de un estudio detallado sobre su diversidad en el país y de una revisión taxonómica global y actualizada. Como resultado, el aprovechamiento y conservación de los integrantes de esta familia es limitado.

Preguntas: ¿Qué avances se han logrado en el estudio y entendimiento de la riqueza, endemismo y distribución de las Lamiaceae mexicanas? ¿Qué estrategias deben ejecutarse para consolidar el conocimiento de la familia en el país?

Taxon: Lamiaceae

Sitio de estudio: México

Métodos: Se hizo una revisión exhaustiva de las Lamiaceae mexicanas en la literatura, bases de datos y herbarios. Se sintetizó el estado de su conocimiento. Se aplicaron análisis cuantitativos para evaluar su riqueza, endemismo y distribución geográfica.

Resultados: México cuenta con 33 géneros y 598 especies, de las cuales el $66.2 \%$ son endémicas. El género más diverso es Salvia, con 306 especies. El estado más diverso es Oaxaca, mientras que Jalisco alberga el mayor número de especies endémicas.

Conclusiones: En México, Lamiaceae es la octava familia más diversa y el número de sus especies representa el $5.5 \%$ de la familia a nivel mundial, por lo que el país puede considerarse uno de los centros de diversificación más importante. Debido a lo anterior, y al endemismo elevado que presenta, México es crucial para la conservación in situ de la familia. Si bien se ha logrado un avance considerable de su conocimiento y en la actualidad hay un repunte en su estudio, es necesario diversificar los campos de investigación.

Palabras clave: distribución geográfica, endemismo, Lamiaceae mexicanas, riqueza florística.

\section{Abstract}

Background: Lamiaceae is one of the most species-rich families in Mexico. However, a detailed treatment on its diversity and an updated comprehensive taxonomic review for the country are still lacking. As a result, the use and conservation of the members of Lamiaceae is limited.

Questions: Which advances have been achieved on the study and understanding of the Mexican Lamiaceae in terms of richness, endemism and distribution? Which strategies should be applied to enhance the knowledge of Lamiaceae in Mexico?

Taxon: Lamiaceae

Study site: Mexico

Methods: A thorough review of Mexican Lamiaceae in the literature, databases and herbaria was conducted. The state of knowledge of the family was summarized. Quantitative analyses were performed to assess its richness, endemism and geographical distribution.

Results: Mexico embraces 33 genera and 598 species of Lamiaceae, with an endemism proportion of $66.2 \%$. The most diverse genus is Salvia, with 306 species. Oaxaca is the state encompassing the highest richness, whereas Jalisco harbors the highest amount of endemic species.

Conclusions: In Mexico Lamiaceae is the eighth most species-rich family, with a number of species equivalent to $5.5 \%$ of all Lamiaceae worldwide; hence the country is one of the most relevant diversification centers for the family. The high species richness and endemism level found in Mexico, makes this country crucial for in situ conservation of the labiates. Currently, subtantial advances have been achieved on the knowledge of Lamiaceae, and their study has increased in recent years. Nervertheless, several fields of research still need to be explored.

Key words: endemism, floristic richness, geographic distribution, Mexican Lamiaceae 


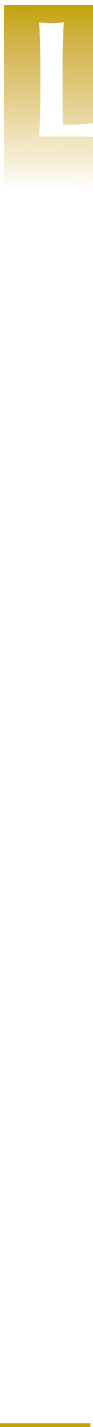

\section{Contribuciones:}

Todos los autores contribuyeron en el curado del listado de Lamiaceae de México, la redacción y revisión del manuscrito.

M. Martínez-Gordillo coordinó, diseño, redacción, compilación y revisión.

B. Bedolla-García y S. Zamudio redacción y compilación de bibliografía.

G. Cornejo-Tenorio y M.R. García-Peña síntesis de datos y redacción.

I. Fragoso-Martínez redacción, compilando la bibliografía relevante y correcciones.

J.G. González-Gallegos organización de la estructura del artículo y redacción.

S. Lara-Cabrera redacción. a familia Lamiaceae, con 236 géneros y 7,173 especies conocidas, se encuentra en el sexto lugar por su riqueza a nivel mundial y es también una de las de mayor riqueza en México (Harley et al. 2004, Figura 1). En su circunscripción actual consiste en un grupo emparentado con Orobanchaceae, Mazaceae, Paulowniaceae, Phrymaceae y Rehmanniaceae (Wagstaff \& Olmstead 1997, Wagstaff et al. 1998, Harley et al. 2004, Marx et al. 2010, Schäferhoff et al. 2010), lo que contrasta con la concepción tradicional de considerarla cercana a Verbenaceae (Cronquist 1981). En la actualidad se reconocen 10 subfamilias dentro de Lamiaceae (Ajugoideae, Cymarioideae, Lamioideae, Nepetoideae, Peronematoideae, Premnoideae, Prostantheroideae, Scutellarioideae, Symphorematoideae y Viticoideae) y cinco clados más inclusivos (Calliprostatherina, Cymalamiina, Perolamiina, Scutelamiina y Viticisymphorina) (Li et al. 2016).

Las Lamiaceae tienen distribución cosmopolita y se encuentran en casi todos los ecosistemas terrestres, aunque son más abundantes en bosques de clima templado, en zonas montañosas. En el mundo existen seis regiones de alta diversidad (Hedge 1992): Mediterráneo y SO de Asia Central, África y Madagascar, China, Australia, Sudamérica y Norteamérica (incluido México).

Lamiaceae es muy diversa en cuanto a su morfología y se distingue porque presenta hojas opuestas, tallos tetrangulares, inflorescencia generalmente compuesta de cimas, rodeadas por brácteas por lo común foliáceas, corolas gamopétalas, en su mayoría bilabiadas, androceo compuesto por 2 o 4 estambres, gineceo bicarpelar y dividido en cuatro lóculos por la intrusión de la pared del ovario, este último súpero, y el fruto, con frecuencia, compuesto por cuatro mericarpos (Harley et al. 2004, Figura 1). Además, muchas de sus especies presentan glándulas epidérmicas que secretan compuestos volátiles aromáticos, por lo que son apreciadas desde el punto de vista culinario (albahaca, menta, tomillo, orégano, romero), medicinal (marrubio, menta, poleo, salvia, toronjil) y dentro de la industria de la perfumería (lavanda, pachuli). Además, debido a lo atractivo de su follaje y flores, se ha promovido su cultivo en muchas regiones del mundo (El-Gazzar \& Watson 1970, Heinrich 1992, Lawrence 1992, Rivera-Núñez \& Obón de Castro 1992, Kintzios 2000, Jenks \& Kim 2013). En particular, existen especies mexicanas que merecen atención por su uso, como la chía (Salvia hispanica L.), por su alto valor nutritivo (Cahill 2003), y la ska pastora (Salvia divinorum Epling \& Játiva), por su efecto psicoactivo (Wasson 1962, Reisfield 1993, Maqueda et al. 2015). Cabe mencionar que el papel de Lamiaceae dentro de las comunidades vegetales es muy relevante dada su interacción con diversos polinizadores y por constituir un elemento, por lo general, abundante o dominante (Huck 1992, Davis et al. 1994, Claßen-Bockhoff 2007, Wester \& Claßen-Bockhoff 2011).

En la República Mexicana, Lamiaceae presenta una riqueza considerable en la mayoría de sus tipos de vegetación; se ha adaptado muy bien a las regiones con climas templados y húmedos, aunque también existe un número significativo de especies que prosperan en zonas secas, con temperaturas relativamente altas. Algunos de sus linajes son endémicos del país o se han diversificado en éste, lo que subyace a la representación amplia de la familia en México, en particular, la de Salvia subgénero Calosphace.

A pesar de su importancia ecológica, evolutiva y económica, y del potencial de usos, la familia todavía no cuenta con un estudio integral y exhaustivo a escala nacional. En contraste, algunas de sus especies pueden estar amenazadas ante los efectos de deterioro ambiental y la pérdida de hábitats, lo que es aún más crítico si se toma en cuenta que el estado de conservación, para la mayoría, no ha sido evaluado. Cabe destacar que sólo dos especies se encuentran en la Norma Oficial Mexicana (SEMARNAT 2010). Por lo anterior, la presente contribución ofrece un diagnóstico de las labiadas en México. A partir de la información generada se discute el avance que se ha logrado en su conocimiento, en qué regiones, disciplinas y complejos taxonómicos hace falta canalizar los esfuerzos futuros y posibles estrategias para promover la formación de recursos humanos en el estudio de Lamiaceae.

\section{Materiales y métodos}

Revisión del conocimiento de Lamiaceae en México. Se realizó una búsqueda exhaustiva de las publicaciones referentes a estudios de diferente índole (sobre todo, taxonómicos y filogenéticos) relacionadas con los géneros existentes en México, a partir de 1800 a la fecha. Se revisaron distintos acervos digitales (Biodiversity Heritage Library, Google Scholar, JSTOR, UNAM en 

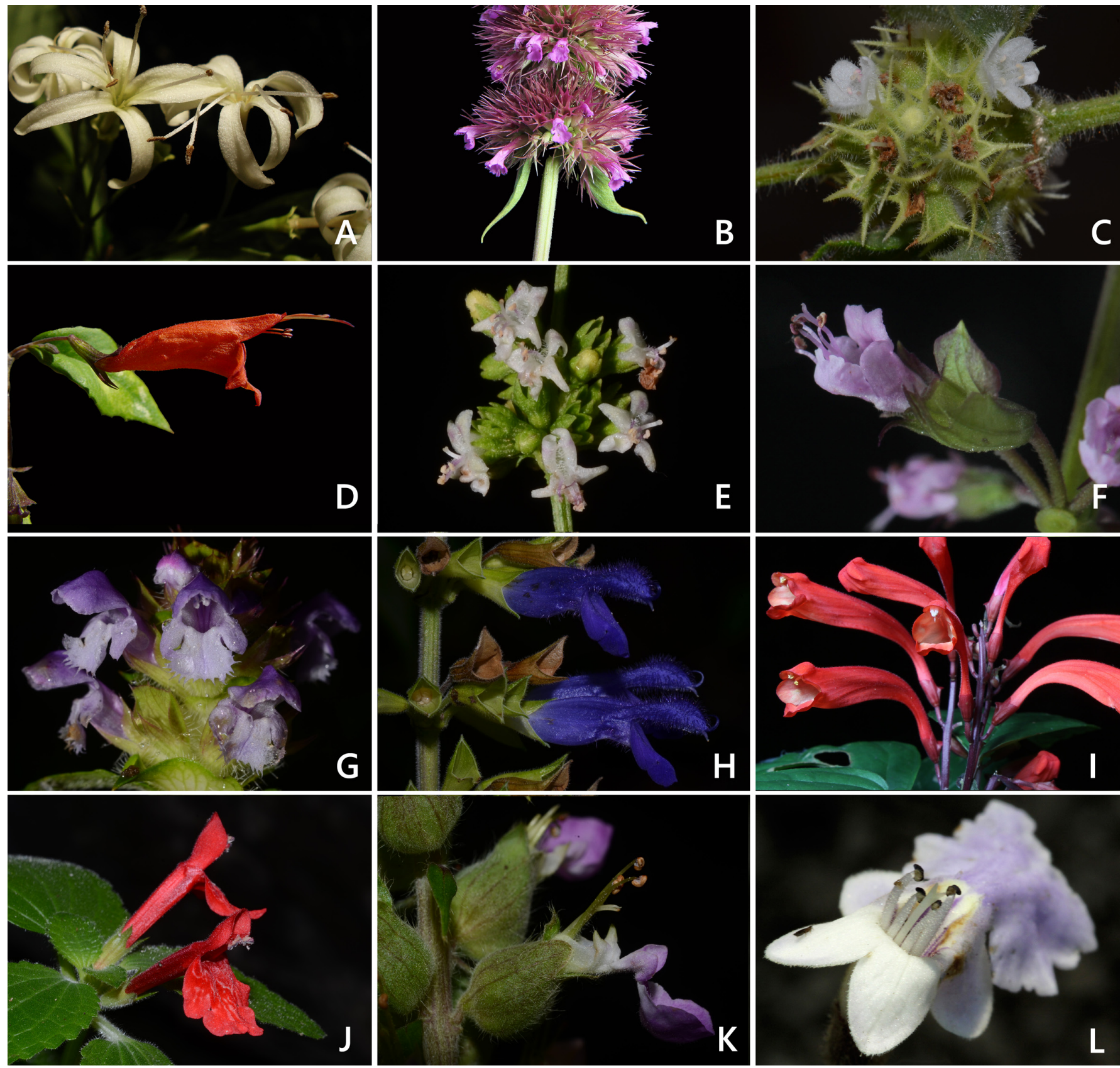

Figura 1. Algunos géneros de Lamiaceae representativos en la flora mexicana. A) Aegiphila (A. costaricensis Moldenke), B) Agastache (A. palmeri (B.L.Rob.) Standl.), C) Asterohyptis (A. stellulata (Benth.) Epling), D) Clinopodium (C. macrostemum (Moc. \& Sessé ex Benth.) Kuntze), E) Hyptis (H. verticillata Jacq.), F) Ocimum (O. carnosum (Spreng.) Link \& Otto ex Benth.), G) Prunella (P. vulgaris L.), H) Salvia (S. mexicana L.), I) Scutellaria (S.formosa Leonard), J) Stachys (S. coccinea Ortega), K) Teucrium (T. vesicarium Mill.) y L) Vitex (V. mollis Kunth). Créditos fotográficos: Guadalupe Cornejo (A, D), Canek Ledesma (B, I), Gerardo Salazar (C, E, G, H, J, K) y Jonathan Amith (F, L).

línea, Wiley Online Library) y bibliotecas nacionales públicas (UNAM) y privadas (Dr. Jerzy Rzedowski Rotter). Una vez concluido lo anterior, se hizo un análisis de la cantidad, actualidad y diversidad de publicaciones disponibles para cada género.

Lista florística. Para la elaboración de la lista florística se recurrió al consenso del criterio taxonómico de los autores, respecto a qué nombres reconocer como correctos, la circunscripción de las especies y su condición como plantas nativas o naturalizadas en México (las especies introducidas y no naturalizadas fueron excluidas del inventario). Se tomó como base la lista aportada por 
Martínez-Gordillo et al. (2013), y en la lista final (Apéndice 1) se incorporaron aquellos nombres aceptados por la mayoría de los autores. Se optó por tal estrategia, dado que la delimitación de especies es compleja y no existen pautas concretas y universales que la regulen (de Queiroz 2005a, b, Hey 2006, Stuessy 2009, Chambers 2012). Por lo tanto, a través de las decisiones consensuadas se obtuvo un inventario actualizado y más robusto de Lamiaceae en México.

Análisis de riqueza, endemismo y distribución. Se consultaron las bases del Herbario CICY (Herbario CICY 2010), Tropicos (2016) y SEINet (2016) y se realizó una amplia consulta de literatura sobre la familia. Las colecciones de herbario consultadas incluyeron a BUAP, CHAP, CIIDIR, ENCB, FCME, IBUG, IEB y MEXU. Con esta información se generó una matriz de presencia de especies de Lamiaceae por entidad federativa en México, a la vez que se verificó si éstas correspondían a especies endémicas del país.

\section{Resultados}

Riqueza florística. Se compiló una lista de 598 especies de Lamiaceae nativas o naturalizadas para México, pertenecientes a 33 géneros y seis subfamilias (Ajugoideae, Lamioideae, Nepetoideae, Premnoideae, Scutellaroideae y Viticoideae), siendo Nepetoideae la más diversa, con 18 géneros (Tabla 1). Los cinco géneros con más especies son: Salvia (306), Scutellaria (61), Stachys (45), Hyptis (35) y Hedeoma (28) (Tabla 1), que en conjunto comprenden casi el $80 \%$ del total de especies de la familia en México, mientras que 11 géneros presentan una sola especie.

Los géneros Acanthomintha, Hypenia y Pogogyne son los únicos que se restringen a una sola entidad en el país, el segundo a Guerrero y los otros dos a Baja California. En cambio, Salvia y Scutellaria se distribuyen en la totalidad de los estados. Del total de especies, 244 (40.8\%) se conocen exclusivamente de un estado y ninguna de las especies se encuentra en todos, siendo la maleza Leonotis nepetifolia (L.) R.Br. la que exhibe mayor distribución (28 de 32).

Endemismo. El 66.2 \% (396 especies) de las 598 especies de Lamiaceae son endémicas de México y pertenecen a 20 géneros. De éstos, los que resguardan la mayor riqueza de especies endémicas y microendémicas (aquellas restringidas a un estado) son: Salvia (236/102, respectivamente), Scutellaria (40/24), Stachys (33/14), Hedeoma (20/11) e Hyptis (16/7) (Tabla 1). Estos cinco géneros abarcan el $87.6 \%$ de las especies endémicas y $88.2 \%$ de las microendémicas. Las especies endémicas de México con áreas de distribución más amplias son Salvia prunelloides Kunth (registrada en 25 estados), S. mexicana L. (23), S. elegans Vahl (20), S. longispicata M. Martens \& Galeotti y Vitex mollis Kunth (18, cada una) (Apéndice 1). Por el contrario, $45 \%$ (179) de las especies son microendémicas (Tabla 1).

Distribución por entidad federativa. Oaxaca reúne la mayor cantidad de géneros (25) y especies (157) nativas o naturalizadas de Lamiaceae (Tabla 2). Esto representa poco más de 75 y $26 \%$ de esos taxa, respectivamente. También resaltan los estados de Puebla, Veracruz y Chiapas, con más de 20 géneros cada uno. Un total de nueve estados superan la cifra de 100 especies (Tabla 2). Si se considera la proporción de especies y la extensión territorial de cada entidad, estos nueve estados también destacan, pero deben considerarse, además, el Estado de México e Hidalgo (Tabla 2). En contraste, los que presentan la menor riqueza son Tlaxcala (18), Baja California Sur (24), Campeche (24), Quintana Roo (24) y Yucatán (26).

Con base en el número de endemismos que albergan, se ubican en orden decreciente, Jalisco (103 especies), Oaxaca (89), Guerrero (86), Puebla (78) y Michoacán (75), mientras que Campeche y Quintana Roo son los únicos en los que no hay especies endémicas (Tabla 2). Jalisco, Oaxaca y Guerrero, también destacan por la presencia de especies microendémicas con 26, 22 y 17 , respectivamente. Es interesante notar que Baja California y Chiapas son los estados que poseen la mayor cantidad de especies exclusivas, es decir, aquéllas que crecen solo en un estado, aunque no califiquen como endémicas (Tabla 2). Un total de siete estados no presentan especies microendémicas ni exclusivas (Aguascalientes, Ciudad de México, Morelos, Tabasco, Tlaxcala, Yucatán y Zacatecas).

Estado del conocimiento en México. El estudio de las Lamiaceae mexicanas ha sido accidentado; no sólo por las dificultades en la circunscripción e identificación de los taxa debido a la elevada similitud morfológica, el reducido polimorfismo molecular y la carencia de tratamientos taxonómicos actuales, sino también por la intermitencia en su abordaje. Han existido períodos 
Tabla 1. Riqueza y endemismo de las especies de Lamiaceae en México. Las especies microendémicas son aquellas cuya distribución se restringe a una sola entidad. Ubicación en la clasificación por subfamilias de acuerdo a Li et al. (2016): ${ }^{1}$ Ajugoideae, ${ }^{2}$ Lamioideae, ${ }^{3}$ Nepetoideae, ${ }^{4}$ Premnoideae, ${ }^{5}$ Scutellaroideae, ${ }^{6}$ Viticoideae, ${ }^{7}$ incertae sedis.

\begin{tabular}{|c|c|c|c|}
\hline Géneros & Especies (\%) & Endémicas (\%) & Microendémicas (\%) \\
\hline Acanthomintha ${ }^{3}$ & $1(0.2)$ & 0 & 0 \\
\hline Aegiphila' & $10(1.7)$ & $1(10.0)$ & $1(10.0)$ \\
\hline Agastache & $13(2.2)$ & 7 (53.9) & $1(7.7)$ \\
\hline Asterohyptis & $2(0.3)$ & $1(50.0)$ & 0 \\
\hline Callicarpa & $1(0.2)$ & 0 & 0 \\
\hline Catoferia & $3(0.5)$ & $1(33.3)$ & $1(33.3)$ \\
\hline Clinopodium $^{3}$ & $14(2.3)$ & $9(64.3)$ & $5(35.7)$ \\
\hline Cornutia ${ }^{4}$ & $1(0.2)$ & $0(0)$ & 0 \\
\hline Cunila & $7(1.2)$ & $6(85.7)$ & $3(42.8)$ \\
\hline Hedeoma & $28(4.6)$ & $20(71.4)$ & $11(39.2)$ \\
\hline Hypenia 3 & $1(0.2)$ & $1(100)$ & 0 \\
\hline Hyptis & $35(5.8)$ & $16(45.7)$ & $7(20.0)$ \\
\hline Leonotis & $1(0.2)$ & 0 & 0 \\
\hline Leonurus ${ }^{2}$ & $1(0.2)$ & 0 & 0 \\
\hline Lepechinia ${ }^{3}$ & $12(2.0)$ & $6(50)$ & $2(16.6)$ \\
\hline Marrubium $^{2}$ & $1(0.2)$ & 0 & 0 \\
\hline Marsypianthes & $1(0.2)$ & 0 & 0 \\
\hline Monarda & $6(1.0)$ & $3(50)$ & $1(16.6)$ \\
\hline Monardella $a^{3}$ & $9(1.5)$ & $2(22.2)$ & $1(11.1)$ \\
\hline Ocimum ${ }^{3}$ & $3(0.5)$ & 0 & 0 \\
\hline Physostegia & $2(0.3)$ & 0 & 0 \\
\hline Pogogyne & $3(0.5)$ & $1(33.3)$ & $1(33.3)$ \\
\hline Poliomintha & $8(1.3)$ & $5(62.5)$ & $3(37.5)$ \\
\hline Prunella3 & $1(0.2)$ & 0 & 0 \\
\hline Salvia3 & $306(51.2)$ & 236 (77.1) & $102(33.3)$ \\
\hline Scutellaria5 & $61(10.2)$ & $40(65.6)$ & $24(39.3)$ \\
\hline Stachys & $45(7.5)$ & $33(73.3)$ & $14(31.1)$ \\
\hline Tetraclea' & $1(0.2)$ & 0 & 0 \\
\hline Teucrium ${ }^{1}$ & $6(1.0)$ & $2(33.3)$ & $1(16.6)$ \\
\hline Trichostema' & $8(1.3)$ & $2(25)$ & $1(12.5)$ \\
\hline Vitex & $4(0.6)$ & $2(50)$ & 0 \\
\hline Volkameria' & $2(0.3)$ & 0 & 0 \\
\hline Warnockia $^{2}$ & $1(0.2)$ & 0 & 0 \\
\hline TOTAL & $598(100)$ & $396(66.2)$ & $179(29.9)$ \\
\hline
\end{tabular}

muy activos, como el impulsado por Epling y colaboradores (1925-1968), quienes realizaron tratamientos y notas para la mayoría de Lamiaceae de América (Mathias 1970, Schmidt-Lebuhn 2006). Posteriormente, siguió un lapso amplio de baja o nula actividad. Después hubo un impulso estimulado por T. P. Ramamoorthy (1983-1993) (Ramamoorthy \& Elliot 1998), para de nuevo pasar a poco más de tres lustros de inactividad. Ahora el estudio de Lamiaceae se encuentra nuevamente en una época de alta actividad de investigación, sobre todo por el descubrimiento de especies nuevas, publicación de tratamientos infra-genéricos y listas florísticas estatales o regionales.

No existe un tratamiento taxonómico que integre la diversidad total de Lamiaceae en el país. A la fecha, se han publicado sólo tres trabajos contemporáneos de tal índole, pero a una escala regional: Flora del Valle de México (Calderón de Rzedowski \& Rzedowski 2001), Flora Mesoamericana (Ulloa-Ulloa et al. 2012) y Revisión taxonómica de Lamiaceae del occidente de México (González-Gallegos et al.2016), que en conjunto representan alrededor del $15 \%$ del territorio continental nacional y cubren entre 40 y $50 \%$ de las especies. También se cuenta con 
Tabla 2. Riqueza, endemismo y restricción de las Lamiaceae en México por entidad federativa. Géneros / Log. (riqueza de géneros / logaritmo natural del área), Especies / Log. (riqueza de especies / logaritmo natural del área (índice de diversidad taxonómica, Squeo et al. 1998)). Las especies exclusivas se restringen a un estado, aunque no son necesariamente endémicas de éste o del país). El área de las entidades se basa en INEGI (2015).

\begin{tabular}{|c|c|c|c|c|c|c|c|}
\hline Estados $\left(\mathbf{k m}^{2}\right)$ & Géneros & Especies & Géneros / Log & Especies / Log & Endémicas & Microendémicas & Exclusivas \\
\hline $\operatorname{AGS}(5,616)$ & 13 & 46 & 1.51 & 5.33 & 18 & 0 & 0 \\
\hline BC $(71,450)$ & 15 & 53 & 1.34 & 4.74 & 13 & 9 & 40 \\
\hline BCS $(73,909)$ & 9 & 24 & 0.8 & 2.14 & 15 & 8 & 8 \\
\hline CAM $(57,508)$ & 11 & 24 & 1 & 2.19 & 0 & 0 & 1 \\
\hline $\mathrm{CHIH}(247,413)$ & 17 & 81 & 1.37 & 6.52 & 31 & 2 & 3 \\
\hline CHIS $(73,311)$ & 21 & 133 & 1.87 & 11.87 & 31 & 8 & 32 \\
\hline $\operatorname{CDMX}(1,495)$ & 12 & 38 & 1.64 & 5.2 & 22 & 0 & 0 \\
\hline COAH $(151,595)$ & 15 & 87 & 1.26 & 7.29 & 41 & 15 & 19 \\
\hline $\operatorname{COL}(5,627)$ & 10 & 47 & 1.16 & 5.44 & 19 & 1 & 1 \\
\hline DGO $(123,364)$ & 18 & 102 & 1.54 & 8.62 & 63 & 9 & 9 \\
\hline GRO $(63,596)$ & 13 & 127 & 1.18 & 11.48 & 85 & 22 & 23 \\
\hline GTO $(30,607)$ & 16 & 71 & 1.55 & 6.87 & 41 & 3 & 3 \\
\hline $\operatorname{HGO}(20,821)$ & 15 & 95 & 1.51 & 9.55 & 54 & 7 & 7 \\
\hline JAL $(78,596)$ & 19 & 151 & 1.69 & 13.4 & 103 & 26 & 26 \\
\hline MÉX $(22,352)$ & 15 & 98 & 1.5 & 9.79 & 62 & 5 & 5 \\
\hline $\mathrm{MICH}(58,599)$ & 17 & 119 & 1.55 & 10.84 & 75 & 6 & 6 \\
\hline MOR $(4,879)$ & 10 & 52 & 1.18 & 6.12 & 31 & 0 & 0 \\
\hline NAY $(27,857)$ & 14 & 74 & 1.37 & 7.23 & 42 & 2 & 2 \\
\hline NL $(64,156)$ & 18 & 103 & 1.63 & 9.31 & 56 & 13 & 14 \\
\hline OAX $(93,757)$ & 25 & 161 & 2.18 & 14.06 & 89 & 17 & 15 \\
\hline PUE $(34,309)$ & 23 & 139 & 2.2 & 13.31 & 77 & 7 & 7 \\
\hline QRO $(11,691)$ & 15 & 78 & 1.6 & 8.33 & 42 & 1 & 1 \\
\hline QROO $(44,706)$ & 13 & 24 & 1.21 & 2.24 & 0 & 0 & 1 \\
\hline $\operatorname{SIN}(57,365)$ & 13 & 61 & 1.19 & 5.57 & 37 & 3 & 4 \\
\hline $\operatorname{SLP}(61,138)$ & 16 & 89 & 1.45 & 8.08 & 47 & 5 & 5 \\
\hline SON $(179,355)$ & 19 & 81 & 1.57 & 6.7 & 31 & 4 & 6 \\
\hline TAB $(24,731)$ & 14 & 33 & 1.38 & 3.26 & 1 & 0 & 0 \\
\hline TAM $(80,249)$ & 16 & 73 & 1.42 & 6.46 & 31 & 2 & 2 \\
\hline $\operatorname{TLX}(3,997)$ & 8 & 17 & 0.96 & 2.05 & 9 & 0 & 0 \\
\hline VER $(71,824)$ & 23 & 124 & 2.06 & 11.09 & 47 & 4 & 4 \\
\hline YUC $(39,524)$ & 13 & 26 & 1.23 & 2.46 & 1 & 0 & 0 \\
\hline ZAC $(75,275)$ & 14 & 64 & 1.25 & 5.7 & 31 & 0 & 0 \\
\hline TOTAL $(\mathbf{1}, 960,672)$ & 33 & 598 & 2.28 & 41.27 & 396 & 179 & 244 \\
\hline
\end{tabular}

listas florísticas para la familia a nivel nacional (Martínez-Gordillo et al. 2013, Villaseñor 2016) y para Chiapas (Domínguez-Vázquez et al. 2002) y Michoacán (Lara-Cabrera et al. 2016).

El género Salvia ha sido el más estudiado en muchos sentidos, por ejemplo, cuenta con una revisión del subgénero Calosphace (Epling 1939a) y en los últimos 20 años se han descubierto y descrito casi 70 especies nuevas para México. También se han realizado revisiones taxonómicas de secciones que incluyen principalmente taxa mexicanos: Axillares (Turner 1996), Audibertia (Epling 1938), Farinaceae (Peterson 1978, Turner 2008), Flocculosae (Turner 2013a), Lavanduloideae (Fragoso-Martínez 2014), Membranaceae (Fragoso-Martínez 2011, GonzálezGallegos 2014), Peninsularis (Turner 2010), Polystachyae (Bedolla-García 2012), Scorodonia (Turner 2009a, Olvera-Mendoza et al. 2017), Sigmoideae (Espejo-Serna \& Ramamoorthy 1993), Standleyana (Turner 2011a) y Uliginosae (Turner 2009b). Se han publicado estudios sobre tipificación de especies (Ramamoorthy 1983, 1984, 1987, Lara-Cabrera \& García-Peña 2008), un catálogo de sinónimos (Alziar 1992) y notas nomenclaturales (González-Gallegos \& Gama-Villanueva 2013, González-Gallegos 2015a), entre otros. Se han logrado avances sustanciales en el entendimiento de sus relaciones filogenéticas (Walker et al. 2004, Walker \& Sytsma 
2007, Jenks et al. 2013, Drew et al. 2017, Fragoso-Martínez et al. 2017a, b). En adición, se han realizado algunos estudios cromosómicos (Epling et al. 1962, Palomino et al. 1986, Mercado et al . 1989), anatómicos (Ceja-Romero et al. 2005, Castro-Morales 2008), genéticos (Cahill 2004), químicos (Wasson 1962, Sánchez et al. 1989, Argumedo et al. 2003), poblacionales (Corral-Bribiesca 2005), florísticos (Cornejo-Tenorio \& Ibarra-Manríquez 2011), etnobotánicos (Reisfield 1993, Dweck 2000, Cahill 2003, 2005, Ayerza \& Coates 2005, Jenks \& Kim 2013) y de interacción planta-insecto (Dieringer et al. 1991, Claßen-Bockhoff et al. 2004, Wester \& Claßen-Bockhoff 2006, Arizmendi et al. 2007, Benitez-Vieyra et al. 2014, Cuevas \& Rosas-Guerrero 2016).

Respecto a Scutellaria, se tiene una revisión taxonómica (Epling 1942) y notas complementarias para las especies americanas (Leonard 1927, Epling 1939b, c, Harley \& Paton 1999), además de una revisión de la sección Resinosa (Turner 1994a), y para la misma, se cuenta con un estudio anatómico de los mericarpos (Turner \& Reveal 2004). En México, la descripción de especies nuevas de Scutellaria se ha dado de manera discontinua (Turner \& Reveal 2004, Cuevas-Guzmán 2010, González-Gallegos \& Vázquez-García 2013). No se ha publicado un análisis filogenético integral de este género con una buena representación de las especies mexicanas.

En Stachys se han realizado dos revisiones taxonómicas: Epling (1934a) y Turner (1994c); la segunda incluye sobre todo especies mexicanas. También se han logrado algunos avances mediante estudios filogenéticos que han incluido varias especies americanas (Lindqvist \& Albert 2002, Roy et al. 2013, 2015, Welch 2016). Se cuenta con la revisión del complejo Stachys coccinea (Turner 1994d, Berumen-Cornejo et al. 2017) y la descripción de especies nuevas ha ocurrido en tiempos recientes (Rzedowski \& García-Zuñiga 1984, Rzedowski \& Calderón, 1988, 1995, Turner 1995a, 2013b, Pool 2007, González-Gallegos 2015b).

Los trabajos publicados para Hyptis incluyen una revisión taxonómica (Epling 1934b, 1949). y varios estudios filogenéticos (El-Gazzar \& Rabei 2008, Harley \& Pastore 2012, Pastore et al. 2011). Para México, se ha descrito un número reducido de especies nuevas (Epling 1939d, González-Gallegos et al. 2014).

Entre las publicaciones de Hedeoma se cuenta con dos revisiones taxonómicas para el género (Epling \& Stewart 1939, Irving 1968, 1980), la descripción de algunas especies mexicanas (Villarreal-Q. 1993, Turner 1991, 1994b, Moran 1999) y de cuatro taxa infraespecíficos (Turner 1991, 1995b). También se encuentran trabajos sobre la prospección de compuestos químicos de especies mexicanas en aplicaciones médicas (Viveros-Valdez et al. 2008, 2010, Leyva-López et al. 2016). No están disponibles análisis filogenéticos amplios o específicos del género.

Clinopodium cuenta con una revisión parcial, bajo el nombre de Satureja, que es más completa con respecto a las especies sudamericanas (Epling 1927, Epling \& Játiva 1964, 1966) y una lista de las especies que deben ser tratadas dentro de su circunscripción (Harley \& Paucar 2000), carece aún de un análisis filogenético robusto.

Agastache cuenta con una revisión taxonómica a nivel genérico (Lint \& Epling 1945) y una más de la sección Brittonastrum (Sanders 1987). La descripción de taxa nuevos del género para el país ha sido relativamente escasa en las últimas décadas con un lapso intermedio de 30 años sin novedades de este tipo (Sanders 1981, Bye et al. 1987, González-Gallegos \& López-Enríquez 2017). Además, se registra un trabajo anatómico y etnobotánico de especies mexicanas (Santillán-Ramírez et al. 2008). También se han realizado algunos estudios para comprender mejor los compuestos que subyacen a las propiedades aromáticas y curativas de sus especies (Hernández-Abreu et al. 2011, Estrada-Reyes et al. 2014, Sánchez-Recillas et al. 2014, FloresFlores et al. 2016) y aquellos que podrían ser útiles en la conservación de granos y vegetales en almacenes (Juárez et al. 2015). Este género aún no cuenta con un análisis que esclarezca sus relaciones filogenéticas.

Lepechinia tiene una sinopsis (Epling 1948), un estudio sistemático contemporáneo (Hart 1983) y una serie de publicaciones que han fundamentado su estatus filogenético y que han ayudado a esclarecer su circunscripción (Drew \& Sytsma 2011, 2013, Drew et al.2014). Dentro de la última década, se han descrito tres especies mexicanas (Martínez-Gordillo \& Lozada-Pérez 2009, Henrickson et al. 2011, Turner 2013c) y se han aportado notas sobre la distribución y filogenia de algunas especies en particular (González-Gallegos et al.2015). Existen algunos avances incipientes de estudios fitoquímicos y de prospección de compuestos activos (Dimayuga et al. 1991, Estrada-Soto et al. 2009, Sánchez-Recillas et al. 2014). 
Asterohyptis es un género básicamente mexicano y cuenta con dos revisiones taxonómicas (Epling 1933, Turner 2011b), al parecer todas sus especies ya han sido descritas. Cunila es un género bastante estudiado, cuenta con una revisión taxonómica reciente (García-Peña 2008), una filogenia preliminar (Agostini et al. 2012), notas nomenclaturales (García-Peña \& Chiang 2003) y descripción de especies nuevas para México (García-Peña 1989, García-Peña \& Tenorio-Lezama 1997, García-Peña \& González-Gallegos 2013). Trichostema cuenta con una monografía (Lewis 1945), una revisión de las especies mexicanas (Lewis \& Rzedowski 1978) y un estudio palinológico (Abu-Asab \& Cantino 1989). Para Monarda existen revisiones taxonómicas (McClinton \& Epling 1946, Scora 1969, Turner 1994e) y un análisis filogenético (Prather et al. 2002); además, su estudio ha abordado enfoques múltiples: evolutivo (Scora 1966b, 1967a, c), filogenético (Huang et al. 2008), fitoquímico (Scora 1966a, 1967b, d) y ecológico con el estudio de las interacciones planta-insecto (Scora 1964). Los géneros Aegiphila (Moldenke 1934), Catoferia (Ramamoorthy 1986), Monardella (Epling 1925), Physostegia (Cantino 1982), Pogogyne (Howell 1931), Poliomintha (Irving 1972) y Teucrium (McClintock \& Epling 1946) han sido poco estudiados o tienen pocos taxa representados en el país, aunque todos cuentan con revisiones taxonómicas. Monardella, además, presenta una nota nomenclatural relacionada con especies mexicanas (Elvin \& Sanders 2009) y en Poliomintha, se tiene la descripción de algunas especies (Epling \& Wiggings 1940, Turner 1993) y trabajos sobre sus constituyentes químicos y sus propiedades (Rivero-Cruz et al. 2011, García-Pérez et al. 2013, Cid-Pérez et al. 2016). De los géneros anteriores, los que cuentan con análisis filogenéticos son Physostegia (Scheen et al. 2008, Roy et al. 2016) y Pogogyne (Silveira \& Simpson 2013). En el caso de Hypenia sólo se ha publicado una especie para México (Martínez-Gordillo \& Valencia-Ávalos 2009). Los géneros Acanthomintha, Callicarpa, Cornutia, Marsypianthes, Ocimum, Leonorus, Leonotis, Marrubium, Tetraclea, Vitex, Volkameria y Warnockia únicamente han sido abordados en algunas floras regionales (ej. Calderón de Rzedowski \& Rzedowski 2001, Berumen-Cornejo 2006, González-Gallegos et al. 2016).

En conjunto, México reúne 33 géneros nativos y/o naturalizados de Lamiaceae, de los cuales el $57.5 \%$ cuenta con tratamientos taxonómicos, aunque la mayoría no están actualizados. Por fortuna, los géneros que no cuentan con revisiones taxonómicas tienen poca representatividad en México, con excepción de Clinopodium, con 14 especies: Acanthomintha (1 especie), Callicarpa (2), Cornutia (1), Marsyphianthes (2), Ocimum (3), Prunella (1), Tetraclea (1), Volkameria (2), Vitex (4) y Warnockia (1).

\section{Discusión}

Riqueza florística y endemismo. Lamiaceae es una de las familias con mayor número de especies en México, sólo superada por Asteraceae, Fabaceae, Orchidaceae, Poaceae, Euphorbiaceae, Rubiaceae y Cactaceae, mientras que Salvia se considera el género más diverso del país (Villaseñor 2016).

En este trabajo se enlistan 598 especies y 33 géneros de Lamiaceae, estas cifras son muy parecidas a las 591 especies y 32 géneros registrados por Martínez-Gordillo et al. (2013) y a las 601 especies y 35 géneros incluidos en Villaseñor (2016). Sin embargo, aunque en la numeralia no existen diferencias significativas, sí hay discrepancias importantes en la identidad de los taxa y nombres incluidos. Por ejemplo, de los 32 géneros reportados por Martínez-Gordillo et al. (2013), Chaunostoma y Neoeplingia son considerados actualmente como sinónimos del género Lepechinia, con base en análisis filogenéticos (Drew et al. 2014). En el presente trabajo se incluyen los géneros Leonurus, Leonotis y Marrubium, porque existen ejemplares de herbario que respaldan su presencia en ambientes silvestres en el país, a diferencia de las listas de Martínez-Gordillo et al. (2013) y de Villaseñor (2016), en donde no incluyen especies naturalizadas. Además de los géneros citados anteriormente, Villaseñor (2016) no considera a Prunella (género naturalizado en el país), pero si a Mentha, del cual no encontramos evidencia de que tenga especies naturalizadas en México. Las especies del género Satureja del Nuevo Mundo son consideradas en este trabajo dentro de Clinopodium (Cantino \& Wagstaff 1998, Harley \& Paucar 2000); en contraste, Villaseñor (2016) enlista especies de ambos géneros; un análisis más detallado respecto a éstas, revela que la única especie de Satureja que reporta es Sature- 
ja sauderi Epling, nombre inexistente, y que más bien corresponde a Satureja ganderi Epling, sinónimo de Clinopodium ganderi (Epling) Govaerts. El mencionado autor también incluye el género Clerodendron; no obstante, no hay representantes de este género naturalizados en México, aunque algunas especies sí se cultivan. Por último, en Villaseñor (2016) se encuentran los géneros Cantinoa, Condea y Mesosphaerum, ya que él sigue la propuesta de Harley \& Pastore (2012) de segregación del género Hyptis, la cual no se acepta en este trabajo, porque se considera que no tiene un soporte robusto, ni morfológica, ni filogenéticamente (González-Gallegos et al. 2014).

La flora vascular de México contiene casi $50 \%$ de especies endémicas (Villaseñor 2016) pero en Lamiaceae el porcentaje es mayor. Ramamoorthy \& Elliott (1998) lo calcularon aproximadamente en $75 \%$ y Martínez-Gordillo et al. (2013) en $65.8 \%$, mientras que en el presente trabajo alcanza el $66.2 \%$. Además, se ha detectado que casi la mitad de las especies endémicas son microendémicas o endémicas a nivel estatal (Tabla 1, Apéndice 1). A nivel de género no hay endemismos en el país, ya que el único descrito como tal, Neoeplingia (Ramamoorthy et al. 1982), fue incorporado a Lepechinia, con base en análisis filogenéticos (Drew et al. 2014). Sin embargo, para siete géneros (e.g., Cunila o Hedeoma), más del $60 \%$ de sus especies son endémicas (Tabla 1).

Distribución por entidades estatales. Los cinco estados con mayor riqueza de especies de Lamiaceae (Tabla 2) coincide, en gran medida, con los registrados por Ibarra-Manríquez et al. (2015) y Villaseñor (2016) respecto a las lianas y a la flora vascular del país. La única discrepancia es la inclusión de Puebla, en lugar de Veracruz. En cambio, es notable que en cuanto al orden, Jalisco y Puebla antecedan a Chiapas. La exclusión de Veracruz y la menor prominencia de la diversidad en Chiapas, puede ser un reflejo de la mayor afinidad de los linajes dominantes de Lamiaceae en México (Nepetoideae, sobre todo Hedeoma, Salvia y Stachys) por áreas de influencia laurásica o neártica y de bosques templados (Hedge 1992, Ramamoorthy \& Elliott 1998); esto también puede explicar, en parte, la pobreza de especies en la Península de Yucatán. Además, para entender la riqueza elevada en Jalisco sería importante considerar la consolidación del Eje Volcánico Transmexicano como una barrera biogeográfica y punto de encuentro entre las biotas de la Sierra Madre Occidental, Sierra Madre Oriental y la Sierra Madre del Sur, lo que habría estimulado procesos de especiación. Patrones e hipótesis similares de alta riqueza en esta entidad se han observado en otros grupos vegetales como Cosmos (Vargas-Amado et al. 2013), Physalis (Zamora-Tavares et al. 2016), y en la tribu Tigridieae de Iridaceae (Munguía-Lino et al. 2015). Por su parte, Puebla atraviesa por una etapa de esclarecimiento de la magnitud de su diversidad gracias a empresas recientes en la exploración de áreas nuevas dentro de su territorio y un esfuerzo más riguroso en el estudio de su flora (Rodríguez-Acosta 2011, Rodríguez-Acosta et al.2014), lo que se corrobora en un incremento superior al $20 \%$ del inventario de sus angiospermas (Rodríguez-Acosta et al. 2014, Villaseñor 2003, 2016, Villaseñor \& Ortiz 2014). La riqueza elevada de labiadas en Puebla puede ser producto de la heterogeneidad fisiográfica que contiene, derivada del encuentro en ella de varias de las provincias biogeográficas (Morrone 2005) y florísticas del país (Rzedowski 1978). No obstante, cabe destacar que lo anterior podría obedecer a un sesgo de la información por entidad, ya que existen recuentos florísticos recientes para Oaxaca (García-Mendoza \& Meave 2011), Jalisco (Ramírez-Delgadillo et al. 2010) y Puebla (Rodríguez-Acosta et al. 2014), además, de un estudio específico que incluye a Jalisco (González-Gallegos et al. 2016). Estudios futuros en otros estados podrían modificar lo discutido previamente.

En contraste a la riqueza global, Baja California y Chiapas resaltan por reunir la mayor cantidad de especies no compartidas con otras entidades del país. Esto puede explicarse por la singularidad de sus biotas y la fuerte influencia de la Provincia Florística de California y la flora de Centroamérica, respectivamente (Wiggins 1980, Klitgaard 2012).

Líneas de investigación prioritarias. Es importante estimular el desarrollo de trabajos florísticos en la familia; se propone que se realicen tratamientos taxonómicos por regiones o por estados, y que los mismos contemplen la recolecta botánica dirigida e intensiva en lugares poco explorados. Para Lamiaceae, es crucial la exploración en los ecotonos de hábitats tropicales y templados en la Sierra Madre Occidental (los sistemas de barrancas en Chihuahua, Durango, Jalisco, Sinaloa y Sonora); las sierras de Cuale, Cacoma y el Halo en Jalisco; la Sierra de Coalcomán en Michoacán; la Sierra Madre del Sur, en las regiones de Costa Grande, Tierra Caliente y Montaña en Guerrero; sierras de Juárez y Huautla, y la Sierra Atravesada en Oaxaca; Sierra Gorda en los 
estados de Guanajuato, Hidalgo, Querétaro y San Luis Potosí; y la Sierra Norte de Puebla. También es necesario que se incopore una mayor cantidad de especies mexicanas y secuencias de ADN en los análisis filogenéticos, a fin de esclarecer sus relaciones evolutivas. En este aspecto, y conforme continúen los avances y la reducción de los costos en campos novedosos como la filogenómica, transcriptómica y secuenciación de genomas completos, las historias evolutivas serán más robustas y estables (e.g., Fragoso-Martínez et al. 2017b).

Existen, además, áreas amplias de oportunidad en que se pueden tomar como modelos las especies de Lamiaceae, ya sea para probar o responder hipótesis específicas, o para contribuir en el bagaje de información sobre ellas. Se pueden tomar en cuenta los estudios de distribución actual y potencial, bajo diferentes escenarios ambientales, el análisis anatómico detallado de caracteres con gran posibilidad de resultar informativos para la filogenia o la taxonomía, análisis genéticos y citológicos que ayuden a comprender mejor los procesos de diversificación de la familia (hibridación, poliploidía), estudios ecológicos para esclarecer las interacciones con el ambiente que actúan como fuerzas que moldean la composición de las especies y los perfiles ambientales óptimos para su crecimiento, la prospección mediante la fitoquímica puede revelar caracteres diagnósticos o sinapomórficos nuevos. Otro aspecto importante es fomentar los estudios etnobotánicos en torno a las labiadas, para que todos estos estudios repercutan en un desarrollo armónico de las comunidades que las aprovechan.

En suma, estas disciplinas y los enfoques interdisciplinarios que puedan emerger de ellas, serían un paso adelante en la estructuración de una monografía de Lamiaceae para el país, y la actualización y estandarización de los tratamientos taxonómicos por géneros o subgrupos de ellos. Los resultados, en conjunto, del abanico de estudios propuestos convergirán en la resolución de problemas taxonómicos y el establecimiento de un sistema de clasificación natural, estable y predictivo.

¿Cómo fomentar la formación de especialistas en la familia? En la actualidad, existe una crisis de la taxonomía que se refleja en el número decreciente de taxónomos (Villaseñor 2015) y el reducido financiamiento para esta labor. La familia Lamiaceae también ha sido afectada por esta crisis, a pesar de su valor ecológico, evolutivo, utilidad y belleza. Actualmente, se cuenta sólo con 10 especialistas nacionales y dos extranjeros, con diversas contribuciones particularmente en Cunila, Hyptis, Lepechinia y Salvia. Sin embargo, se debe incrementar el número de especialistas en otros géneros, especialmente aquellos que incluyen un alto número de especies endémicas y microendémicas (Tabla 1).

El interés de las nuevas generaciones por el estudio de las plantas es cada día menor, por diferentes causas, entre ellas la falta de reconocimiento del trabajo taxonómico (Wandersee \& Schussler 1999, 2001). Para revertir esta situación es importante fomentar la formación de especialistas en la familia y difundir entre la sociedad la importancia de la sistemática como una disciplina científica, rigurosa y precisa, que estudia a la biodiversidad. También se sugiere incrementar la presencia en foros de especialistas de los diferentes campos de estudio de Lamiaceae, donde los estudiantes puedan intercambiar opiniones y experiencias, como sucede en el Congreso Mexicano de Botánica, y se involucren en proyectos de investigación y de difusión. En este ámbito es importante la gestión de recursos para ofrecer becas y apoyos a quienes se dediquen a esta disciplina.

Conocer conlleva entender y apreciar, por lo que se debe estimular la elaboración para Lamiaceae de manuales, guías de campo, profusamente ilustrados, de nivel regional, estatal o de áreas naturales protegidas y crear una página web (con diagnosis, claves, fotos, además de los saberes tradicionales, usos, horticultura, novedades en investigación, líneas prometedoras a desarrollar, directorio de especialistas, organización de excursiones, concursos y exposiciones). Estas actividades deben promoverse y ser valoradas como producto derivado de la investigación taxonómica y florística, cuyos resultados puedan ser compartidos con comunidades rurales y urbanas; además de valorarse en las evaluaciones de productividad de los investigadores.

\section{Agradecimientos}

Agradecemos a Guillermo Ibarra Manríquez, Presidente de la Sociedad Botánica Mexicana (2014-2016), por impulsarnos a realizar el presente trabajo, así como por sus atinadas correc- 
ciones a la versión final del manuscrito; a los curadores de los herbarios por las facilidades otorgadas en la consulta de los ejemplares, al editor de la revista Botanical Sciences y a los revisores del manuscrito por sus valiosas sugerencias. Apreciamos también el apoyo de Daniel Le (The Field Museum Herbarium), Christine Niezgoda (The Field Museum Herbarium), Rebecca Peters (California Academy of Sciences), James C. Solomon (Missouri Botanical Garden) y Debra Trock (California Academy of Sciences), quienes nos proporcionaron imágenes digitalizadas de especímenes de herbario cuya determinación y distribución era dudosa. Agradecemos a Gerardo Salazar, Canek Ledesma y Jonathan Amith por facilitarnos fotografías de las especies representativas de Lamiaceae.

\section{Literatura citada}

Abu-Asab MS, Cantino PD. 1989. Pollen morphology of Trichostema (Labiatae) and its systematic implications. Systematic Botany 14: 359-369. DOI: 10.2307/2418926

Agostini G, Echeverrigaray S, Souza-Chies TT. 2012. A preliminary phylogeny of the genus Cunila D. Royen ex L. (Lamiaceae) based on ITS rDNA and trnL-F regions. Molecular Phylogenetics and Evolution 65: 739-747. DOI: 10.1016/j.ympev.2012.07.030

Alziar G. 1992. Catalogue synonymique des Salvia du monde (Lamiaceae): 5. Biocosme Mesogéen 9: 413-497.

Argumedo DR, Parra-Delgado H, Ramírez-Apan MT, Nieto-Camacho A, Martínez-Vázquez M. 2003. Isolation and chemical transformations of some anti-inflammatory triterpenes from Salvia mexicana L. var. minor Benth. Journal of the Mexican Chemical Society 47: 167-172.

Arizmendi MC, Monterrubio-Solís C, Juárez L, Flores-Moreno I, López-Saut E. 2007. Effect of the presence of nectar feeders on the breeding success of Salvia mexicana and Salvia fulgens in a suburban park near México City. Biological Conservation 136: 155-158. DOI: 10.1016/j.biocon.2006.11.016

Ayerza R, Coates W. 2005. Chia, rediscovering a forgotten crop of the Aztecs. Tucson: The University of Arizona Press.

Bedolla-García BY. 2012. Filogenia de Salvia secc. Polystachyae (Lamiaceae). PhD Thesis, Universidad Michoacana de San Nicolás de Hidalgo.

Benitez-Vieyra S, Fornoni J, Pérez-Alquicira J, Boege K, Domínguez CA. 2014. The evolution of signalreward correlations in bee- and hummingbird-pollinated species of Salvia. Proceedings of the Royal Society B 281: 20132934. DOI: 10.1098/rspb.2013.2934

Berumen-Cornejo AM. 2006. Tratado florístico de la familia Lamiaceae Martinov (excepto Salvia L.) para el estado de Aguascalientes, México. MSc. Thesis, Universidad Nacional Autónoma de México.

Berumen-Cornejo AM, Charlotte L, Pérez Molphe Balch EM, Siqueiros Delgado ME. 2017. Phylogeny of the Stachys coccinea (Lamiaceae) complex based on molecular and morphological data. Systematic Botany 42: 484-493. DOI 10.1600/036364417X696113

Bye R, Linares E, Ramamoorthy TP, García F, Collera O, Palomino G, Corona V. 1987. Agastache mexicana subsp. xolocotziana (Lamiaceae), a new taxon from the Mexican medicinal plants. Phytologia 62: 157-163.

Cahill JP. 2003. Ethnobotany of Chia, Salvia hispanica L. (Lamiaceae). Economic Botany 57: 604-618. DOI: 10.1663/0013-0001(2003)057[0604:EOCSHL]2.0.CO;2

Cahill JP. 2004. Genetic diversity among varieties of Chia (Salvia hispanica L.). Genetic Resources and Crop Evolution 51: 773-781. DOI: 10.1023/B:GRES.0000034583.20407.80

Cahill JP. 2005. Human selection and domestication of chia (Salvia hispanica L.). Journal of Ethnobiology 25: 155-174. DOI: 10.2993/0278-0771(2005)25[155:HSADOC]2.0.CO;2

Calderón de Rzedowski G, Rzedowski J. 2001. Flora fanerogámica del Valle de México. Pátzcuaro, Michoacán: Instituto de Ecología, A.C., Comisión Nacional para el Conocimiento y Uso de la Biodiversidad.

Cantino PD. 1982. A monograph of the genus Physostegia (Labiatae). Contributions from the Gray Herbarium of Harvard University 211: 1-105.

Cantino PD, Wagstaff SJ. 1998. A reexamination of North American Satureja s.l. in light of molecular evidence. Brittonia 50: 63-70. DOI: 10.2307/2807719

Castro-Morales M. 2008. Caracterización morfológica del cáliz, polen y fruto de Salvia secc. Polystachyae (Lamiaceae). BSc. Thesis, Universidad Michoacana de San Nicolás de Hidalgo.

Ceja-Romero J, Pérez-Olvera CP, Rivera-Tapia J. 2005. Anatomía de la madera de las especies mexicanas de Salvia sección Erythrostachys Epl. (Lamiaceae). Boletín de la Sociedad Botánica de México 76: 53-59.

Chambers G. 2012. The species problem: seeking new solutions for philosophers and biologists. Biology \& Philosophy 27: 755-765. DOI: 10.1007/s10539-012-9314-6

Cid-Pérez TS, Torres-Muñoz JV, Nevárez-Moorillón GV, Palou E, López-Malo A. 2016. Chemical charac- 
terization and antifungal activity of Poliomintha longiflora Mexican oregano. Journal of Essential Oil Research 28: 157-165. DOI: 10.1080/10412905.2015.1083490

Claßen-Bockhoff R. 2007. Floral construction and pollination biology in the Lamiaceae. Annals of Botany 100: 359-360. DOI: $10.1093 / \mathrm{aob} / \mathrm{mcm} 157$

Claßen-Bockhoff R, Speck T, Tweraser E, Wester P, Thimm S, Reith M. 2004. The stamina lever mechanism in Salvia L. (Lamiaceae): a key innovation for adaptive radiation? Organisms, Diversity \& Evolution 4: 189-205. DOI: 10.1016/j.ode.2004.01.004

Cornejo-Tenorio G, Ibarra-Manríquez G. 2011. Diversidad y distribución del género Salvia (Lamiaceae) en Michoacán, México. Revista Mexicana de Biodiversidad 82: 1279-1296.

Corral-Bribiesca JI. 2005. Variación morfológica y molecular de RAPD's en Salvia polystachya (Lamiaceae). BSc. Thesis, Universidad Michoacana de San Nicolás de Hidalgo.

Cronquist A. 1981. An integrated system of classification of flowering plants. New York: Columbia University Press.

Cuevas E, Rosas-Guerrero V. 2016. Spatio-temporal variation of nectar robbing in Salvia gesneriflora and its effects on nectar production and legitimate visitors. Plant Biology 18: 9-14. DOI: 10.1111/plb.12311

Cuevas-Guzmán R. 2010. Scutellaria sipilensis (Lamiaceae), una especie nueva para el occidente de México. Novon 20: 396-399. DOI: 10.3417/2009035

Davis FW, Stine PA, Stoms DM. 1994. Distribution and conservation status of coastal sage scrub in southwestern California. Journal of Vegetation Science 5: 743-756. DOI: 10.2307/3235887

de Queiroz K. 2005a. A unified concept of species and its consequences for the future taxonomy. Proceedings of the California Academy of Sciences 56: 196-215.

de Queiroz K. 2005b. Different species problems and their resolution. BioEssays 27: 1263-1269.

Dieringer G, Ramamoorthy TP, Tenorio LP. 1991. Floral visitors and their behavior to sympatric Salvia species (Lamiaceae) in Mexico. Acta Botánica Mexicana 13: 75-83. DOI: 10.21829/abm13.1991.609

Dimayuga RE, Garcia SK, Nielsen PH, Christophersen C. 1991. Traditional medicine of Baja California Sur (Mexico). III. Carnosol: a diterpene antibiotic from Lepechinia hastata. Journal of Ethnopharmacology 31: 43-48. DOI: 10.1016/0378-8741(91)90142-Z

Domínguez-Vázquez G, Berlin B, Castro-Ramírez AE, Estrada-Lugo EIJ. 2002. Revisión de la diversidad y patrones de distribución de Labiatae en Chiapas. Anales del Instituto de Biología, Serie Botánica 73: 39-80.

Drew BT, Cacho NI, Sytsma KJ. 2014. The transfer of two rare monotypic genera, Neoeplingia and Chaunostoma, to Lepechinia (Lamiaceae), and notes of their conservation. Taxon 63: 831-842. DOI: $10.12705 / 634.6$

Drew BT, Sytsma KJ. 2011. Testing the monophyly and placement of Lepechinia in the tribe Mentheae (Lamiaceae). Systematic Botany 36: 1038-1049. DOI: 10.1600/036364411X605047

Drew BT, Sytsma KJ. 2013. The South American radiation of Lepechinia (Lamiaceae): phylogenetics, divergence times and evolution of dioecy. Botanical Journal of the Linnean Society 171: 171-190. DOI: 10.1111/j.1095-8339.2012.01325.x

Drew BT, González-Gallegos JG, Xiang C-L, Kriebel R, Drummond CP, Walker JB, Sytsma KJ. 2017. Salvia united: The greatest good for the greatest number. Taxon 66: 133-145. DOI: $10.12705 / 661.7$

Dweck AC. 2000. The folklore and cosmetic use of various Salvia species. In: Kintzios E, ed. Sage: the Genus Salvia. Amsterdam: Overseas Publishers Association, 1-25.

El-Gazzar A, Watson L. 1970. Some economic implications of the taxonomy of Labiatae essential oils and rusts. New Phytologist 69: 487-492. DOI: 10.1111/j.1469-8137.1970.tb02444.x

El-Gazzar A, Rabei S. 2008. Taxonomic assessment of five numerical methods and its implications on the classification of Hyptis s.1. (Labiatae). International Journal of Botany 4: 85-92. DOI: 10.3923/ ijb.2008.85.92

Elvin MA, Sanders AC. 2009. Nomenclatural changes for Monardella (Lamiaceae) in California. Novon 19: $315-343$. DOI: $10.3417 / 2008044$

Epling C. 1925. Monograph of the genus Monardella. Annals of the Missouri Botanical Garden 12: 1-106. DOI: $10.2307 / 2394581$

Epling C. 1927. Studies on South American Labiatae III. Synopsis of the genus Satureia. Annals of the Missouri Botanical Garden 14: 47-86.

Epling C. 1933. Asterohyptis: a newly proposed genus of Mexico and Central America. Bulletin of the Torrey Botanical Club 60: 17-21. DOI: 10.2307/2480486

Epling C. 1934a. Preliminary revision of American Stachys. Repertorium Specierum Novarum Regni Vegetabilis 80: 1-75.

Epling C. 1934b. Synopsis of the genus Hyptis in North America. Repertorium Specierum Novarum Regni Vegetabilis 34: 73-130.

Epling C. 1938. The Californian salvias: a review of Salvia, section Audibertia. Annals of the Missouri Botanical Garden 25: 95-188. 
Epling C. 1939a. A revision of Salvia subgenus Calosphace. Repertorium Specierum Novarum Regni Vegetabilis 110: 1-383.

Epling C. 1939b. Apuntes sobre el género Scutellaria de la América tropical y subtropical. Lilloa 4: 229-275.

Epling C. 1939c. Notes on the Scutellariae of western North America. Madroño 5: 49-72.

Epling C. 1939d. Two Mexican species of Hyptis. Madroño 5: 15-16.

Epling C. 1942. The American species of Scutellaria. University of California Publications in Botany 20: $1-137$.

Epling C. 1948. A synopsis of the Tribe Lepechinieae (Labiatae). Brittonia 6: 352-364.

Epling C. 1949. Revisión del género Hyptis (Labiatae). Revista del Museo de La Plata (Nueva Serie), Sección Botánica 7: 153-497.

Epling C, Játiva C. 1964. Revisión del género Satureja en América del Sur. Brittonia 16: 393-416.

Epling C, Játiva C. 1966. A descriptive key to the species of Satureja indigenous to North America. Brittonia 18: 244-248.

Epling C, Lewis PH, Raven H. 1962. Chromosomes of Salvia: section Audibertia. Aliso 5: 217-221.

Epling C, Stewart WS. 1939. A revision of Hedeoma with a review of allied genera. Repertorium Specierum Novarum Regni Vegetabilis 115: 1-49.

Epling C, Wiggins IL. 1940. A new Poliomintha from Baja California. Contributions from the Dudley Herbarium 3: 85-86.

Espejo-Serna A, Ramamoorthy TP. 1993. Revisión taxonómica de Salvia sección Sigmoideae (Lamiaceae). Acta Botánica Mexicana 23: 65-102.

Estrada-Reyes R, López-Ruvalcava C, Ferreyra-Cruz OA, Dorantes-Barrón AM, Heinze G, Moreno-Aguilar J, Martínez-Vázquez M. 2014. Central nervous system effects and chemical composition of two subspecies of Agastache mexicana; an ethnomedicine of Mexico. Journal of Ethnopharmacology 153: 98-110. DOI: 10.1016/j.jep.2013.12.057

Estrada-Soto S, Arteaga-González V, Castillo-España P, Molina-Salinas GM, Said-Fernández S, Navarrete-Vázquez G. 2009. Anti-mycobacterial activity of extracts from plants used in Mexico for the treatment of infectious diseases. Pharmacognosy Magazine 5: 106-110.

Flores-Flores A, Hernández-Abreu O, Ríos MY, León-Rivera I, Aguilar-Guadarrama B, Castillo-España P, Perea-Arango I, Estrada-Soto S. 2016. Vasorelaxant mode of action of dichloromethane-soluble extract from Agastache mexicana and its main bioactive compounds. Pharmaceutical Biology 54: 2807-2813. DOI: $10.1080 / 13880209.2016 .1184690$

Fragoso-Martínez I. 2011. Revisión taxonómica de la sección Membranaceae del género Salvia en México. BSc. Thesis, Universidad Nacional Autónoma de México.

Fragoso-Martínez I. 2014. Análisis filogenético del complejo Salvia lavanduloides Kunth (Lamiaceae). MSc. Thesis, Universidad Nacional Autónoma de México.

Fragoso-Martínez I, Martínez-Gordillo M, Salazar GA, Sazatornil F, Jenks AA, García-Peña MR, BarreraAveleida G, Benitez-Vieyra S, Magallón S, Cornejo-Tenorio G, Granados-Mendoza C. 2017a. Phylogeny of the Neotropical sages (Salvia subg. Calosphace; Lamiaceae) and insights into pollinator and area shifts. Plant Systematics and Evolution DOI 10.1007/s00606-017-1445-4

Fragoso-Martínez I, Salazar GA, Martínez-Gordillo M, Magallón S, Sánchez-Reyes L, Lemmon EM, Lemmon AR, Sazatornil F, Granados-Mendoza C. 2017b. A pilot study applying the plant Anchored Hybrid Enrichment method to New World sages (Salvia subgenus Calosphace; Lamiaceae). Molecular Phylogenetics Evolution DOI: 10.1016/j.ympev.2017.02.006

García-Mendoza AJ, Meave JA. 2011. Diversidad florística de Oaxaca: de musgos a angiospermas. Ciudad de México: Universidad Nacional Autónoma de México y Comisión Nacional para el Conocimiento y Uso de la Biodiversidad.

García-Peña MR. 1989. A new species of Cunila (Lamiaceae) from southwestern Mexico. Brittonia 44: 727-730. DOI: $10.2307 / 4110438$

García-Peña MR. 2008. Revisión taxonómica del género Cunila (Lamiaceae) en Norteamérica y Centroamérica. MSc. Thesis, Universidad Nacional Autónoma de México.

García-Peña MR, Chiang F. 2003. Cunila leucantha and C. polyantha (Lamiaceae), confused neotropical species. Taxon 52: 129-132. DOI: $10.2307 / 3647312$

García-Peña MR, González-Gallegos JG. 2013. Cunila jaliscana (Lamiaceae) a new species from Jalisco, Mexico. Phytotaxa 125: 17-24. DOI: 10.11646/phytotaxa.125.1.3

García-Peña MR, Tenorio-Lezama P. 1997. Especie nueva de Cunila (Lamiaceae) del estado de Durango, México. Anales del Instituto de Biología de la Universidad Nacional Autónoma de México, Serie Botánica 68: 1-5.

García-Pérez E, Noratto GD, García-Lara S, Gutiérrez-Uribe JA, Mertens-Talcott SU. 2013. Micropropagation effect on the anti-carcinogenic activity of polyphenolics from Mexican oregano (Poliomintha glagrescens Gray) in human colon cancer cells HT-29. Plant Foods for Human Nutrition 68: 155-162. DOI: $10.1007 / \mathrm{s} 11130-013-0344-2$ 
González-Gallegos JG. 2014. Revision of Salvia subg. Calosphace sect. Membranaceae (Lamiaceae). Telopea 16: 43-81. DOI: 10.7751/telopea20147483

González-Gallegos JG. 2015a. Salvia ramamoorthyana and S. omissa (Lamiaceae), two names for two old and largely confused species from Mexico. Phytotaxa 236: 215-225. DOI: 10.11646/phytotaxa.236.3.2

González-Gallegos JG. 2015b. Stachys harkerae (Lamioideae, Lamiaceae), a new species from Western Mexico. Annales Botanici Fennici 52: 345-351. DOI: 10.5735/085.052.0512

González-Gallegos JG, Gama-Villanueva OJ. 2013. Resurrection of Salvia species (Lamiaceae) recently synonymized in Flora Mesoamericana. Phytotaxa 151: 1-24. DOI: 10.11646/phytotaxa.151.1.1

González-Gallegos JG, Vázquez-García JA. 2013. Scutellaria cuevasiana and Scutellaria sublitoralis (Lamiaceae), two new species from Jalisco and Nayarit, Mexico. Revista Mexicana de Biodiversidad 84: 20-29. DOI: $10.7550 / \mathrm{rmb} .28434$

González-Gallegos JG, Drew B, Cuevas-Guzmán R. 2015. Novelties on the distribution of Lepechinia flammea (Lamiaceae), rediscovery of L. glomerata in Jalisco, Mexico, and their phylogenetic position within Lepechinia. Botanical Sciences 93: 1-14. DOI: 10.17129/botsci.125

González-Gallegos JG, Castro-Castro A, Flores-Argüelles A, Romero-Guzmán AR. 2014. Discovery of Hyptis pseudolantana in Jalisco and Michoacán, and description of H. cualensis and H. macvaughii (Ocimeae, Lamiaceae), two new species from western Mexico. Phytotaxa 163: 149-165. DOI: 10.11646/phytotaxa.163.3.2

González-Gallegos JG, Castro-Castro A, Quintero-Fuentes V, Mendoza-López ME, De Castro-Arce E. 2016. Revisión taxonómica de Lamiaceae del occidente de México. Ibugana 7: 3-345.

González-Gallegos JG, López-Enríquez IL. 2017. Agastache sandersiana (Lamiaceae): a new species from northwestern Durango, Mexico. The Journal of the Torrey Botanical Society 144: 97-103. DOI: 10.3159/TORREY-D-15-00057.1

Harley RM, Atkins S, Budantsev AL, Cantino PD, Conn BJ, Grayer R, Harley MM, de Kok R, Krestovskaya T, Morales R, Paton AJ, Ryding O, Upson T. 2004. Labiatae. In: Kubitzki JW, ed. The Families and Genera of Vascular Plants 7. Flowering Plants. Dicotyledons. Lamiales (Except Acanthaceae including Avicenniaceae).: 167-275.

Harley RM, Pastore JFB. 2012. A generic revision and new combinations in the Hyptidinae (Lamiaceae), based on molecular and morphological evidence. Phytotaxa 58: 1-55. DOI: 10.11646/phytotaxa.58.1.1

Harley RM, Paton A. 1999. Notes on new world Scutellaria. Kew Bulletin 54: 221-225. DOI: $10.2307 / 4111044$

Harley RM, Paucar AG. 2000. List of species of tropical American Clinopodium (Labiatae), with new combinations. Kew Bulletin 55: 917-927. DOI: 10.2307/4113638

Hart JA. 1983. Systematic and evolutionary studies in the genus Lepechinia (Lamiaceae) PhD Thesis, Harvard University.

Hedge IC. 1992. A global survey of the biogeography of the Labiatae. In: Harley RM, Reynolds T, eds. Advances in Labiatae Science. Kew: Royal Botanic Gardens, 7-17.

Heinrich M. 1992. Economic botany of American Labiatae. In: Harley RM, Reynolds T, eds. Advances in Labiatae Science. Kew: Royal Botanic Gardens, 475-488.

Henrickson J, Fishbein M, Van Devender TR. 2011. Lepechinia yecorana (Lamiaceae), a new dioecious species from the Yécora area of Sonora, Mexico. Journal of the Botanical Research Institute of Texas 5: $67-74$

Herbario CICY. 2010. Flora de la península de Yucatán. <http://www.cicy.mx/sitios/flora\%20digital/>. (accessed may, 2016).

Hernández-Abreu O, Durán-Gómez L, Best-Brown R, Villalobos-Molina R, Rivera-Leyva J, Estrada-Soto S. 2011. Validated liquid chromatographic method and analysis of content of tilianin on several extracts obtained from Agastache mexicana and its correlation with vasorelaxant effect. Journal of Ethnopharmacology 138: 487-491. DOI: 10.1016/j.jep.2011.09.041

Hey J. 2006. On the failure of modern species concepts. Trends in Ecology and Evolution 21: 447-450. DOI: $10.1016 /$ j.tree.2006.05.011

Howell JT. 1931. The genus Pogogyne. Proceedings of the California Academy of Sciences 4th series 20: 105-128. DOI: 10.5962/bhl.part.24282

Huang M, Crawford DJ, Freudenstein JV, Cantino PD. 2008. Systematics of Trichostema (Lamiaceae): evidence from ITS, ndhF, and morphology. Systematic Botany 33: 437-446. DOI: 10.1600/036364408 784571554

Huck RB. 1992. Overview of pollination biology in the Labiatae. In: Harley RM, Reynolds T, eds. Advances in Labiatae Science. Kew: Royal Botanic Gardens, 167-181.

Ibarra-Manríquez G, Rendón-Sandoval FJ, Cornejo-Tenorio G, Carrillo-Reyes P. 2015. Lianas of Mexico. Botanical Sciences 93: 365-417. DOI:10.17129/botsci.123 
INEGI 2015. Panorama sociodemográfico de México 2015. <http://www3 inegi.org.mx/sistemas/Panorama2015/Web/Webinicial.aspx> (accessed january, 2017).

Irving RS. 1968. Systematics of Hedeoma (Labiatae). PhD Thesis, University of Texas.

Irving RS. 1972. A revision of the genus Poliomintha (Labiatae). Sida 5: 8-22.

Irving RS. 1980. The systematics of Hedeoma (Labiatae). Sida 8: 218-295.

Jenks AA, Kim SC. 2013. Medicinal plant complexes of Salvia subgenus Calosphace: an ethnobotanical study of new world sages. Journal of Ethnopharmacology 146: 214-224. DOI: 10.1016/ j.jep.2012.12.035

Jenks AA, Walker JB, Kim SC. 2013. Phylogeny of New World Salvia subgenus Calosphace (Lamiaceae) based on cpDNA (psbA-trnH) and nrDNA (ITS) sequence data. Journal of Plant Research 126: 483 496. DOI: $10.1007 / \mathrm{s} 10265-012-0543-1$

Juárez ZN, Hernández LR, Bach H, Sánchez-Arreola E, Bach H. 2015. Antifungal activity of essential oils extracted from Agastache mexicana ssp xolocotziana and Porophyllum linaria against post-harvest pathogens. Industrial Crops and Products 74: 178-182. DOI: 10.1016/j.indcrop.2015.04.058

Kintzios SE. 2000. Sage: the genus Salvia. Amsterdam: Harwood Academic Publishers.

Klitgaard, B. 2012. Salvia L. In: Davidse G, Sousa M, Knapp S, Chiang F. eds Flora Mesoamericana 4(2), Rubiaceae a Verbenaceae. Saint Louis: Missouri Botanical Press, 396-424.

Lara-Cabrera SI, García-Peña MR. 2008. Lectotypification of Salvia elegans Vahl. (Lamiaceae). Revista Mexicana de Biodiversidad 79: 261-264.

Lara-Cabrera SI, Bedolla-García BY, Zamudio S, Domínguez-Vázquez G. 2016. Diversidad de Lamiaceae en el estado de Michoacán, México. Acta Botánica Mexicana 116: 107-149.

Lawrence BM. 1992. Chemical components of Labiatae oils and their exploitation. In: Harley RM, Reynolds T, eds. Advances in Labiatae Science. Kew: Royal Botanic Gardens, 399-436.

Leonard EC. 1927. The North American species of Scutellaria. Contributions from the United States National Herbarium 22: 703-748.

Lewis H. 1945. A revision of the genus Trichostema. Brittonia 5: 276-303.

Lewis H, Rzedowski J. 1978. The genus Trichostema (Labiatae) in Mexico. Madroño 25: 151-154.

Leyva-López N, Nair V, Bang WY, Cisneros-Zevallos L, Heredia JB. 2016. Protective role of terpenes and polyphenols from three species of oregano (Lippia graveolens, Lippia palmeri and Hedeoma patens) on the supression of lipopolysaccharide-induced inflammation in RAW 264.7 macrophage cells. Journal of Ethnopharmacology 187: 302-312. DOI: 10.1016/j.jep.2016.04.051

Li B, Cantino PD, Olmstead RG, Bramley GLC, Xiang C-L, Ma Z-H, Tan Y-H, Zhang D-X. 2016. A largescale chloroplast phylogeny of the Lamiaceae sheds new light on its subfamilial classification. Scientific Reports 6: 34343 . DOI: $10.1038 /$ srep34343

Lindqvist C, Albert VA. 2002. Origin of the Hawaiian endemic mints within North American Stachys (Lamiaceae). American Journal of Botany 89: 1709-1724. DOI: 10.3732/ajb.89.10.1709

Lint H, Epling C. 1945. A Revision of Agastache. American Midland Naturalist 33: 207-208. DOI: $10.2307 / 2421328$

Maqueda AE, Valle M, Addy PH, Antonijoan RM, Puntes M, Coimbra J, Ballester MR, Garrido M, González M, Claramunt J, Barker S, Johnson MW, Griffiths RR, Riba J. 2015. Salvinorin-A induces intense dissociative effects, blocking external sensory perception and modulatin interoception and sense of body ownership in humans. International Journal of Neuropsychopharmacology 18: 1-14. DOI: 10.1093/ijnp/pyv065

Martínez-Gordillo M, Fragoso-Martínez I, García-Peña MR, Montiel O. 2013. Géneros de Lamiaceae de México, diversidad y endemismo. Revista Mexicana de Biodiversidad 84: 30-86. DOI: 10.7550/ rmb.30158

Martínez-Gordillo M, Lozada-Pérez L. 2009. Una especie nueva de Lepechinia sección Glomeratae (Lamiaceae) de México. Brittonia 61: 112-115. DOI: 10.1007/s12228-008-9070-8

Martínez-Gordillo M, Valencia-Ávalos S. 2009. Una nueva especie de Hypenia (Lamiaceae) de México. Brittonia 61: 67-70. DOI: 10.1007/s12228-008-9069-1

Mathias ME. 1970. Carl Clawson Epling (1894-1968). Taxon 19: 88-91.

Marx HE, O’Leary N, Yuan Y-W, Lu-Irving P, Tank DC, Múlgura ME, Olmstead RG. 2010. A molecular phylogeny and classification of Verbenaceae. American Journal of Botany 97: 1647-1663. DOI: 10.3732/ajb.1000144

McClintock E, Epling C. 1946. A revision of Teucrium in the New World, with observations on its variation, geographical distribution and history. Brittonia 5: 491-510.

Mercado P, Ramamoorthy TP, Palomino G. 1989. Karyotypes of five Mexican species of Salvia subgenus Calosphace (Lamiaceae). Cytologia 54: 605-608. DOI: 10.1508/cytologia.54.605

Moldenke HN. 1934. A monograph of the genus Aegiphila. Brittonia 1: 245-262.

Moran R. 1999. Hedeoma matomianum (Labiatae), a new species from Baja California, Mexico. Madroño 46: $212-214$. 
Morrone JJ. 2005. Hacia una síntesis biogeográfica de México. Revista Mexicana de Biodiversidad 76: 207-252.

Munguía-Lino G, Vargas-Amado G, Vázquez-García LM, Rodríguez A. 2015. Riqueza y distribución geográfica de la tribu Tigridieae (Iridaceae) en Norte América. Revista Mexicana de Biodiversidad 86: 80-98. DOI: $10.1038 /$ srep34343

Olvera-Mendoza EI, Bedolla-García BY, Lara-Cabrera SI. 2017. Revisión taxonómica de Salvia subgénero Calosphace sección Scorodoniae (Lamiaceae), endémica de México. Acta Botánica Mexicana 118: 7-39. DOI: $10.21829 / \mathrm{abm} 118.2017 .1198$

Palomino G, Mercado P, Ramamoorthy TP. 1986. Chromosomes of Salvia subgenus Calosphace (Lamiaceae), a preliminary report. Cytologia 51: 381-386. DOI: 10.1508/cytologia.51.381

Pastore JFB, Harley RM, Forest F, Paton A, van den Berg C. 2011. Phylogeny of the subtribe Hyptidinae (Lamiaceae tribe Ocimeae) as inferred from nuclear and plastid DNA. Taxon 60: 1317-1329.

Peterson KM. 1978. Systematic studies of Salvia L. subgenus Calosphace (Benth.) Benth. in Benth. \& Hook. section Farinaceae (Epling) Epling (Lamiaceae). PhD Thesis, University of Maryland.

Pool A. 2007. New species of Stachys (Lamiaceae) from Mesoamerica. Novon 17: 60-66. DOI: 10.3417/1055-3177(2007)17[60:NSOSLF]2.0.CO;2

Prather LA, Monfils AK, Posto AL, Williams RA. 2002. Monophyly and phylogeny of Monarda (Lamiaceae): evidence from the internal transcribed spacer (ITS) region of nuclear ribosomal DNA. Systematic Botany 27: 127-137. DOI: 10.1043/0363-6445-27.1.127

Ramamoorthy TP. 1983. Lectotypification of Salvia spp. (Labiatae) described by Cavanilles. Taxon 32: 637-638.

Ramamoorthy TP. 1984. Typifications in Salvia (Lamiaceae). Taxon 33: 322-324. DOI: 10.2307/1221181

Ramamoorthy TP. 1986. A revision of Catoferia (Labiatae). Kew Bulletin 41: 299-305. DOI: $10.2307 / 4102933$

Ramamoorthy TP. 1987. Neotypification of Mexican Salvia pulchella (Lamiaceae). Taxon 36: 135-136.

Ramamoorthy TP, Elliot M. 1998. Lamiaceae de México: diversidad, distribución, endemismo y evolución. In: Ramamoorthy TP, Bye R, Lot A, Fa J, eds. Diversidad Biológica de México: Orígenes y Distribución. México: Instituto de Biología, Universidad Nacional Autónoma de México, 501-525.

Ramamoorthy TP, Hiriart-Valencia P, González-Medrano F. 1982. Neoplingia Ramamoorthy, Hiriart \& Medrano (Labiatae) un nuevo género de Hidalgo, México. Boletín de la Sociedad Botánica de México 43: 61-65.

Ramírez-Delgadillo R, Vargas-Ponce O, Arreola-Nava HJ, Cedano-Maldonado M, González-Tamayo R, González-Villarreal LM, Harker M, Hernández-López L, Martínez-González RE, Pérez de la Rosa JA, Rodríguez-Contreras A, Reynoso-Dueñas JJ, Villarreal de Puga LM, Villaseñor-Ríos JL. 2010. Catálogo de plantas vasculares de Jalisco. Guadalajara: Universidad de Guadalajara, Sociedad Botánica de México, Universidad Autónoma Metropolitana.

Reisfield AS. 1993. The botany of Salvia divinorum (Labiatae). Sida 15: 349-366.

Rivera-Núñez D, Obón de Castro C. 1992. The ethnobotany of Old World Labiatae. In: Harley RM, Reynolds T, eds. Advances in Labiatae Science. Kew: Royal Botanic Gardens, 455-473.

Rivero-Cruz I, Duarte G, Navarrete A, Bye R, Linares E, Mata R. 2011. Chemical composition and antimicrobial and spasmolytic properties of Poliomintha longiflora and Lippia graveolens essential oils. Journal of Food Science 76: 309-317. DOI: 10.1111/j.1750-3841.2010.02022.x.

Rodríguez-Acosta M. 2011. Diversidad vegetal. In: Comisión Nacional para el Conocimiento y Uso de la Biodiversidad (CONABIO), La Biodiversidad en Puebla, Estudio de Caso. México, D.F.: CONABIO, Gobierno del Estado de Puebla, Benemérita Universidad Autónoma de Puebla, 119-138.

Rodríguez-Acosta M, Villaseñor JL, Coombes AJ, Cerón-Carpio AB. 2014. Flora del estado de Puebla, México. Puebla: Benemérita Universidad Autónoma del Estado de Puebla.

Roy T, Catlin NS, Garner DMG, Cantino PD, Scheen A-C, Lindqvist C. 2016. Evolutionary relatioships within the lamioid tribe Synandreae (Lamiaceae) based on multiple low-copy nuclear loci. PeerJ 4: e2220. DOI: $10.7717 /$ peerj.2220

Roy T, Chang TH, Lan T, Lindqvist C. 2013. Phylogeny and biogeography of New World Stachydeae (Lamiaceae) with emphasis on the origin and diversification of Hawaiian and South American taxa. Molecular Phylogenetics and Evolution 69: 218-138. DOI: 10.1016/j.ympev.2013.05.023

Roy T, Cole LW, Chang TH, Lindqvist C. 2015. Untangling reticulate evolutionary relationships among New World and Hawaiian mints (Stachydeae, Lamiaceae). Molecular Phylogenetics and Evolution 89: 46-62. DOI: 10.1016/j.ympev.2015.03.023

Rzedowski J. 1978. Provincias florísticas de México. In: Rzedowski J. Vegetación de México. México, D.F.: Limusa, 104-121.

Rzedowski J, Calderón de Rzedowski G. 1988. Tres especies nuevas de Stachys (Labiatae) de México. Acta Botánica Mexicana 3: 1-5. 
Rzedowski J, Calderón de Rzedowski G. 1995. Tres adiciones a la flora fanerogámica de México. Acta Botánica Mexicana 32: 1-10. DOI: 10.21829/abm32.1995.741

Rzedowski J, García-Zúñiga A. 1984. Una nueva especie de Stachys (Labiatae) del centro de México. Phytologia 56: 321-326.

Sánchez C, Cárdenas J, Rodríguez-Hahn L, Ramamoorthy TP. 1989. Abietane diterpenoids of Salvia anastomosans. Phytochemistry 28: 1681-1684. DOI: 10.1016/S0031-9422(00)97824-5

Sánchez-Recillas A, Mantecón-Reyes P, Castillo-España P, Villalobos-Molina R, Ibarra-Barajas M, Estrada-Soto S. 2014. Tracheal relaxation of five medicinal plants used in Mexico for the treatment of several diseases. Asian Pacific Journal of Tropical Medicine 7: 179-183. DOI: 10.1016/S19957645(14)60017-1

Sanders RW. 1981. New Taxa and combinations in Agastache (Lamiaceae). Brittonia 33: 194-197. DOI: $10.2307 / 2806322$

Sanders RW. 1987. Taxonomy of Agastache section Brittonastrum (Lamiaceae-Nepeteae). Systematic Botany Monographs 15: 1-92. DOI: 10.2307/25027677

Santillán-Ramírez MA, López-Villafranco ME, Aguilar-Rodríguez S, Aguilar-Contreras A. 2008. Estudio etnobotánico, arquitectura foliar y anatomía vegetativa de Agastache mexicana spp. mexicana y A. mexicana ssp. xolocotziana. Revista Mexicana de Biodiversidad 79: 513-524.

Schäferhoff B, Fleishmann A, Fisher E, Albach DC, Brosh T, Heubl G, Müller KF. 2010. Towards resolving Lamiales relationships: insights from rapidly evolving chloroplast sequences. Evolutionary Biology 10: 352 . DOI: $10.1186 / 1471-2148-10-352$

Scheen AC, Lindqvist C, Fossdal CG, Albert VA. 2008. Molecular phylogenetics of tribe Synandreae, a North American lineage of lamioid mints (Lamiaceae). Cladistics 24: 299-314. DOI: 10.1111/j.10960031.2007.00180.x

Schmidt-Lebuhn AN. 2006. Bibliography of Carl Clawson Epling's taxonomical and floristic works on the genera of New World Labiatae. Candollea 61: 71-82.

Scora RW. 1964. Dependency of pollination on patterns in Monarda (Labiatae). Nature 204: 1011-1012. DOI:10.1038/2041011a0

Scora RW. 1966a. Problems in chemotaxonomy - influence of varying soil conditions of geographical and individual variants upon the distribution of certain substances in chromatographed extracts of Monarda fistulosa. Plant and Soil 24: 145-152. DOI: 10.1007/BF01373080

Scora RW. 1966b. The evolution of the genus Monarda (Labiatae). Evolution 20: 185-190. DOI: 10.1111/ j.1558-5646.1966.tb03354.x

Scora RW. 1967a. Divergence in Monarda (Labiatae). Taxon 16: 499-505. DOI: 10.2307/1216953

Scora RW. 1967b. Essential oil synthesis in Monarda punctata. Lloydia 30: 236-241.

Scora RW. 1967c. Interspecific relationships in the genus Monarda (Labiatae). University of California Publications in Botany 41: 1-71.

Scora RW. 1967d. Study of the essential leaf oils of the genus Monarda (Labiatae). American Journal of Botany 54: 446-452.

Scora RW. 1969. El género Monarda en México. Boletín de la Sociedad Botánica de México 30: 31-71.

SEMARNAT (Secretaría del Medio Ambiente y Recursos Naturales). 2010. Norma Oficial Mexicana NOM-059-SEMARNAT-2010, Protección ambiental - Especies nativas de México de flora y fauna silvestres - Categorías de riesgo y especificaciones para su inclusión, exclusión o cambio - Lista de especies en riesgo. Diario Oficial de la Federación.

Silveira MA, Simpson MG. 2013. Phylogenetic systematics of the mesa mints: Pogogyne (Lamiaceae). Systematic Botany 38: 782-794. DOI: 10.1600/036364413X670313

Squeo FA, Cavieres LA, Arancio G, Novoa JE, Matthei O, Marticorena C, Rodríguez R, Arroyo MTK, Muñoz M. 1998. Biodiversidad de la flora vascular en la Región de Antofagasta, Chile. Revista Chilena de Historia Natural 71:571-591.

Stuessy TF. 2009. Plant taxonomy, the systematic evaluation of comparative data. New York: Columbia University Press.

Tropicos 2016. Missouri Botanical Garden. <http://www.tropicos.org> (accesed april and may, 2016).

SEINet. 2016. Southwest Environmental Information Network, - Arizona Chapter 2009- <http//:swbiodiversity.org/seinet/index.php > (accesed may and june, 2016).

Turner BL. 1991. Novelties and new combinations in Mexican Hedeoma (Lamiaceae). Phytologia 71: $32-$ 37. DOI: 10.5962/bhl.part.16697

Turner BL. 1993. Two new species of Poliomintha (Lamiaceae) from northeastern Mexico. Phytologia 74: 164-167.

Turner BL. 1994a. A taxonomic overview of Scutellaria, section Resinosa (Lamiaceae). Phytologia 76: 345-382. DOI: 10.5962/bhl.part.9962

Turner BL. 1994b. Hedeoma rzedowskii (Lamiaceae), a new species from northcentral Mexico. Phytologia 76: 277-280. DOI: 10.5962/bhl.part.12480 
Turner BL. 1994c. Synopsis of Mexican and Central American species of Stachys (Lamiaceae). Phytologia 77: 338-377. DOI: 10.5962/bhl.part.5571

Turner BL. 1994d. Taxonomic study of the Stachys coccinea (Lamiaceae) complex. Phytologia 76: 391 401.

Turner BL. 1994e. Taxonomic treatment of Monarda (Lamiaceae) for Texas and Mexico. Phytologia 77: 56-79.

Turner BL. 1995a. A new species of Stachys (Lamiaceae) from the Sierra de Manantlán of Jalisco, Mexico. Phytologia 78: 209-210.

Turner BL. 1995b. Two new varieties of Hedeoma palmeri (Lamiaceae) from northeastern Mexico. Phytologia 79: 47-50.

Turner BL. 1996. Synopsis of section Axillaris of Salvia. Phytologia 81: 16-21.

Turner BL. 2008. Recension of Salvia sect. Farinaceae (Lamiacae). Phytologia 90: 163-175.

Turner BL. 2009a. Recension of the Mexican species of Salvia (Lamiaceae), section Scorodonia. Phytologia 91: 256-269.

Turner BL. 2009b. Recension of the Mexican species of section Uliginosae of Salvia (Lamiaceae). Phytologia 91: 440-466.

Turner BL. 2010. Recension of the Mexican species of Salvia (Lamiaceae), sect. Peninsularis. Phytologia 92: 20-26.

Turner BL. 2011a. Recension of Mexican species of Salvia sect. Standleyana (Lamiaceae). Phytoneuron 23: $1-6$.

Turner BL. 2011b. Overview of the genus Asterohyptis (Lamiaceae) and description of a new species from northern Mexico. Phytoneuron 2: 1-6.

Turner BL. 2013a. Taxonomic overview of the Mexican species of Salvia sect. Flocculosae (Lamiaceae). Phytoneuron 36: 1-11.

Turner BL. 2013b. Mexican species of Stachys (Lamiaceae) revisited. Phytologia 95: 27-33.

Turner BL. 2013c. A new species of Lepechinia (Lamiaceae) from Oaxaca, Mexico. Phytologia 95: 138-140.

Turner BL, Reveal JL. 2004. A new species of Scutellaria (Lamiaceae) from Guerrero, Mexico. Sida 21: 679-681.

Ulloa-Ulloa C, Davidse G, Sousa SM, Knapp S, Chiang F. 2012. Flora Mesoamericana Vol. 4, Parte 2. Rubiaceae a Verbenaceae. St. Louis: Universidad Nacional Autónoma de México, Missouri Botanical Garden, The Natural History Museum (London).

Vargas-Amado G, Castro-Castro A, Harker M, Villaseñor JL, Ortiz E, Rodríguez A. 2013. Distribución geográfica y riqueza del género Cosmos (Asteraceae: Coreopsidae). Revista Mexicana de Biodiversidad 84: 536-555. DOI: $10.7550 / \mathrm{rmb} .31481$

Villaseñor J. 2003. Diversidad y distribución de las Magnoliophyta de México. Interciencia 28: 160-167.

Villaseñor J. 2015. ¿La crisis de la biodiversidad es la crisis de la taxonomía? Botanical Sciences 93: 3-14. DOI: $10.17129 /$ botsci. 456

Villaseñor JL, Ortiz E. 2014. Biodiversidad de las plantas con flores (División Magnoliophyta) en México. Revista Mexicana de Biodiversidad 85: 134-142. DOI: 10.7550/rmb.31987

Villaseñor JL. 2016. Checklist of the native vascular plants of Mexico. Revista Mexicana de Biodiversidad 87: 559-902. DOI: $10.1016 /$ j.rmb.2016.06.017

Villarreal-Q JA. 1993. A new species of Hedeoma (Lamiaceae) from northwestern Mexico. Sida 15: 415 418.

Viveros-Valdez E, Rivas-Morales C, Carranza-Rosales P, Mendoza S, Schmeda-Hirschmann G. 2008. Free radical scavengers from the Mexican herbal tea "poleo" (Hedeoma drummondii). Zeitschrift für Naturforschung C 63: 341-346. DOI: 10.1515/znc-2008-5-606

Viveros-Valdez E, Rivas-Morales C, Oranday-Cárdenas A, Castro-Garza J, Carranza-Rosales P. 2010. Antiproliferative effect from the Mexican poleo (Hedeoma drummondii). Journal of Medicinal Food 13: 740-742. DOI: $10.1089 / \mathrm{jmf} .2009 .0041$

Wagstaff SJ, Olmstead RG. 1997. Phylogeny of Labiatae and Verbenaceae inferred from rbcL sequences. Systematic Botany 22: 165-179.

Wagstaff SJ, Hickerson L, Spangler R, Reeves PA, Olmstead RG. 1998. Phylogeny in Labiatae s. 1., inferred from cpDNA sequences. Plant Systematics and Evolution 209: 265-274. DOI: 10.1007/BF00985232

Walker JB, Sytsma KJ. 2007. Staminal evolution in the genus Salvia (Lamiaceae): molecular phylogenetic evidence for multiple origins of the stamina lever. Annals of Botany 100: 375-391. DOI: 10.1093/aob/ mcl176

Walker JB, Sytsma KJ, Treutlein J, Wink M. 2004. Salvia (Lamiaceae) is not monophyletic: implications for the systematic, radiation, and ecological specializations of Salvia and tribe Mentheae. American Journal of Botany 91: 1115-1125. DOI: 10.3732/ajb.91.7.1115

Wandersee JH, Schussler EE. 1999. Preventing plant blindness. The American Biology Teacher 61: 84-86. DOI: $10.2307 / 4450624$ 
Wandersee JH, Schussler EE. 2001. Toward a theory of plant blindness. Plant Science Bulletin 47: 2-9.

Wasson RG. 1962. A new mexican psychotropic drug from the mint family. Botanical Museum Leaflets 20: $77-84$.

Welch AJ, Collins K, Ratan A, Drautz-Moses DI, Schuster SC, Lindqvist C. 2016. The quest to resolve recent radiations: plastid phylogenomics of extinct and endangered Hawaiian endemic mints (Lamiaceae). Molecular Phylogenetics and Evolution 99: 16-33. DOI: 10.1016/j.ympev.2016.02.024

Wester P, Claßen-Bockhoff R. 2006. Hummingbird pollination in Salvia haenkei (Lamiaceae) lacking the typical lever mechanism. Plant Systematics and Evolution 257: 133-146. DOI: 10.1007/s00606-0050366-9

Wester P, Claßen-Bockhoff R. 2011. Pollination syndromes of New World Salvia species with special reference to bird pollination. Annals of the Missouri Botanical Garden 98: 101-155. DOI: $10.3417 / 2007035$

Wiggins I. 1980. Flora of Baja California. Stanford: Stanford University Press.

Zamora-Tavares MP, Mungía-Lino G, Martínez M, Rodríguez-Contreras A, Vargas-Ponce O. 2016. Species richness, distribution, and endemism of subtribe Physalinae (Solanaceae) at different regionalization criteria. In: Zamora-Tavares MP. Relaciones Filogenéticas de la Subtribu Physalinae (Solanaceae) con Énfasis en las Especies Americanas. PhD Thesis, Universidad de Guadalajara, 38-93. 
Apéndice 1. Lista de especies de la familia Lamiaceae en México (las especies endémicas están señaladas con un asterisco). Entre paréntesis, para los géneros, se provee el número de especies totales, endémicas y microendémicas; la distribución por estados se indica para las especies. Abreviaturas: Aguascalientes (AGS), Baja California (BC), Baja California Sur (BCS), Campeche (CAM), Ciudad de México (CDM), Chiapas (CHIS), Chihuahua (CHIH), Coahuila (COAH), Colima (COL), Durango (DGO), Estado de México (MÉX), Guanajuato (GTO), Guerrero (GRO), Hidalgo (HGO), Jalisco (JAL), Michoacán (MICH), Morelos (MOR), Nayarit (NAY), Nuevo León (NL), Oaxaca (OAX), Puebla (PUE), Querétaro (QRO), Quintana Roo (QROO), San Luis Potosí (SLP), Sinaloa (SIN), Sonora (SON), Tabasco (TAB), Tamaulipas (TAM), Tlaxcala (TLX), Veracruz (VER), Yucatán (Yuc), Zacatecas (ZAC).

\section{Acanthomintha (1/1/1)}

Acanthomintha ilicifolia A.Gray. (BC)

\section{Aegiphila (10/1/1)}

Aegiphila costaricensis Moldenke (CHIS, TAB, VER)

Aegiphila deppeana Steud. (CHIS, NAY, OAX, PUE, TAB, VER)

Aegiphila elata Sw. (CHIS, OAX, TAB, VER)

Aegiphila falcata Donn.Sm. (CHIS)

Aegiphila fasciculata Donn.Sm. (CHIS)

Aegiphila monstrosa Moldenke (CAM, CHIS, OAX, QROO, TAB, VER, YUC)

Aegiphila panamensis Moldenke (CHIS)

Aegiphila skutchii Moldenke (CHIS, JAL)

Aegiphila valerioi Standl. (CHIS)

*Aegiphila wigandioides Lundell (CHIS)

Agastache (13/7/1)

*Agastache aurantiaca (A.Gray) Lint \& Epling (CHIH, DGO) Agastache breviflora (A.Gray) Epling (CHIH, SON)

*Agastache coccinea (Greene) Lint \& Epling (CHIH, DGO)

*Agastache eplingiana R. W. Sanders (DGO)

Agastache mearnsii Wooton \& Standl. (CHIH, SON)

*Agastache mexicana (Kunth) Lint \& Epling (CDM, GTO, HGO, MÉX, MICH, PUE, QRO, VER)

Agastache micrantha (A.Gray) Wooton \& Standl. $(\mathrm{CHIH}, \mathrm{COAH}$, DGO, SON)

Agastache pallida (Lindl.) Cory (CHIH, DGO, SON)

Agastache pallidiflora (A.Heller) Rydb. (COAH, NL, SON)

*Agastache palmeri (B.L.Rob.) Standl. (COAH, GTO, HGO, JAL, MÉX, NL, OAX, PUE, QRO, SLP, VER)

*Agastache pringlei (Briq.) Lint \& Epling (CHIH, DGO)

*Agastache sandersiana J.G.González (CHIH, DGO)

Agastache wrightii (Greenm.) Wooton \& Standl. (CHIH, SON)

Asterohyptis (2/1/0)

*Asterohyptis seemannii (A.Gray) Epling (CHIH, DGO, JAL, NAY, SIN, SON)

Asterohyptis stellulata (Benth.) Epling (AGS, CHIH, CHIS, COL, DGO, GRO, GTO, JAL, MÉX, MICH, MOR, NAY, OAX, PUE, SIN, SON, VER, ZAC)

Callicarpa (1/0/0)

Callicarpa acuminata Kunth (CAM, CHIS, JAL, MÉX, NAY, NL, OAX, PUE, QRO, QROO, SLP, TAB, TAM, VER, YUC)

Catoferia (3/1/1)

Catoferia capitata (Benth.) Hemsl. (CHIS, OAX, VER)

Catoferia chiapensis A.Gray ex Benth. (CHIS)

* Catoferia martinezii Ramamoorthy (GRO)

Clinopodium (14/9/5)

*Clinopodium amissum (Epling \& Játiva) Harley (DGO, SIN)
Clinopodium brownei (Sw.) Kuntze (BCS, CHIS, HGO, JAL, NL, PUE, QRO, SIN, SLP, SON, TAB, TAM, VER, YUC)

Clinopodium chandleri (Brandegee) P.D.Cantino \& Wagstaff (BC)

Clinopodium ganderi (Epling) Govaerts (BC)

*Clinopodium hintoniorum (B.L.Turner) Govaerts (NL)

*Clinopodium jaliscanum (McVaugh \& R.Schmid) Govaerts (JAL)

Clinopodium ludens (Shinners) A.Pool (QROO, YUC)

*Clinopodium macrostemum (Moc. \& Sessé ex Benth.) Kuntze (CDM, DGO, GRO, GTO, HGO, JAL, MÉX, MICH, MOR, OAX, PUE, SIN, VER)

*Clinopodium maderense (Henrickson) Govaerts (COAH)

*Clinopodium mexicanum (Benth.) Govaerts (CHIS, GRO, GTO, HGO, OAX, PUE, QRO, SLP, TAM, VER)

*Clinopodium micromerioides (Hemsl.) Govaerts (SLP)

*Clinopodium palmeri (A.Gray) Kuntze (BC)

*Clinopodium procumbens (Greenm.) Harley (CHIS, HGO, OAX, PUE, VER)

Clinopodium selerianum (Loes.) Govaerts (CHIS)

Cornutia (1/0/0)

Cornutia pyramidata L. (CAM, CHIS, OAX, PUE, QROO, TAB, VER, YUC)

Cunila (7/6/3)

* Cunila crenata García-Peña \& Tenorio (DGO)

* Cunila jaliscana García-Peña \& J.G.González (JAL)

Cunila leucantha Kunth ex Schltdl. \& Cham. (CHIS, GRO, OAX, VER)

*Cunila lythrifolia Benth. (CDM, GRO, GTO, HGO, MÉX, MICH, MOR, PUE, QRO, TLX, VER)

*Cunila polyantha Benth. (AGS, COL, DGO, GRO, GTO, JAL, MICH, ZAC)

*Cunila pycnantha B.L.Rob. \& Greenm. (CHIS, COL, DGO, GRO, GTO, JAL, MÉX, MICH, MOR, NAY, OAX, SIN)

* Cunila ramamoorthiana García-Peña (GRO)

Hedeoma (28/20/11)

Hedeoma acinoides Scheele (COAH, NL)

*Hedeoma bella (Epling) R.S.Irving (JAL)

*Hedeoma chihuahuensis (Henrickson) B.L.Turner $(\mathrm{CHIH}$, $\mathrm{COAH})$

*Hedeoma ciliolata (Epling \& W.S.Stewart) R.S.Irving (NL)

Hedeoma costata A.Gray (CHIH, CHIS, COAH, DGO, HGO, NL, OAX, PUE, QRO, SLP, SON, TAM, ZAC)

Hedeoma dentata Torr. (CHIH, SON)

Hedeoma drummondii Benth. (COAH, HGO, NL, QRO, SLP, SON, TAM)

* Hedeoma floribunda Standl. (CHIH, SON) 
Apéndice 1. Continuación.

Hedeoma hyssopifolia A.Gray $(\mathrm{CHIH}, \mathrm{COAH}, \mathrm{SON})$

*Hedeoma irvingii B.L.Turner (COAH, NL)

*Hedeoma johnstonii R.S.Irving (COAH)

*Hedeoma jucunda Greene (DGO)

* Hedeoma martirensis Moran (BC)

* Hedeoma matomiana Moran (BC)

*Hedeoma microphylla R.S.Irving (SLP)

*Hedeoma montana Brandegee $(\mathrm{COAH})$

Hedeoma nana (Torr.) Briq. (AGS, CHIH, COAH, DGO, GTO, $\mathrm{HGO}, \mathrm{NL}, \mathrm{SLP}, \mathrm{SON}, \mathrm{TAM}, \mathrm{ZAC})$

*Hedeoma oblatifolia Villarreal $(\mathrm{CHIH}, \mathrm{COAH})$

Hedeoma oblongifolia (A.Gray) A.Heller (CHIH, SON)

*Hedeoma palmeri Hemsl. (COAH, GTO, HGO, NL, QRO, SLP, TAM)

*Hedeoma patens M.E.Jones (CHIH, DGO, JAL, NAY, SIN, SON, ZAC)

*Hedeoma patrina W.S. Stewart (AGS, COAH, NL, SLP, ZAC)

*Hedeoma piperita Benth. (CDM, HGO, JAL, MÉX, MICH, MOR, PUE)

Hedeoma plicata Torr. (AGS, CHIH, COAH, DGO, JAL, NL, SLP, ZAC)

*Hedeoma pusilla (R.S.Irving) R.S.Irving (NL)

*Hedeoma quercetorum Epling (NL, TAM)

*Hedeoma tenuiflora Brandegee (BC)

*Hedeoma tenuipes Epling (TAM)

Hypenia (1/1/0)

*Hypenia violacea Mart.Gord. \& S.Valencia (GRO, OAX)

Hyptis (35/16/7)

Hyptis albida Kunth (AGS, BC, BCS, CHIH, COL, DGO, GTO, HGO, JAL, MICH, NAY, OAX, PUE, QRO, SIN, SLP, SON,

TAM, VER, ZAC)

* Hyptis anitae Epling \& Játiva (BC, BCS)

Hyptis atrorubens Poit. (CHIS, OAX, PUE, TAB, VER)

Hyptis brevipes Poit. (CHIS, GRO, OAX, PUE, TAB, VER)

Hyptis capitata Jacq. (CAM, CHIS, COL, DGO, GRO, JAL, MICH, NAY, OAX, PUE, QROO, SIN, SLP, TAB, VER, YUC)

*Hyptis collina Brandegee (BCS)

Hyptis conferta Pohl ex Benth. (CHIS, OAX, TAB, VER)

*Hyptis cualensis J.G.González \& Art.Castro (JAL)

*Hyptis decipiens M.E.Jones (BCS)

Hyptis emoryi Torr. (BC, BCS, SON)

Hyptis intermedia Epling (CHIS)

*Hyptis involucrata Benth. (MÉX)

* Hyptis iodantha Epling (GRO)

*Hyptis laniflora Benth. (BCS, SON)

Hyptis lantanifolia Poit. (CHIS, NAY, VER)

*Hyptis macvaughii J.G.González \& Art.Castro (JAL, NAY)

* Hyptis mixta Epling (OAX)

Hyptis mutabilis (Rich.) Briq. (AGS, CAM, CHIH, CHIS, COL,

DGO, GRO, GTO, HGO, JAL, MÉX, MICH, MOR, NAY, NL, OAX, PUE, QRO, QROO, SIN, SLP, SON, TAB, TAM, VER, YUC, ZAC)
Hyptis oblongifolia Benth. (COL, GRO, JAL, MÉX, MICH, NAY, OAX, SIN, TAB, ZAC)

Hyptis pectinata (L.) Poit. (CAM, CHIS, COL, GRO, HGO, JAL, MÉX, MICH, NAY, OAX, PUE, QROO, SLP, SON, TAB, TAM, VER, YUC)

*Hyptis pinetorum Epling (JAL)

*Hyptis pseudolantana Epling (GRO, JAL, MICH)

Hyptis pulegioides Pohl ex Benth. (CHIS)

Hyptis recurvata Poit. (CHIS, VER)

*Hyptis rhytidea Benth. (DGO, JAL, NAY, SIN)

Hyptis scandens Epling (CAM)

*Hyptis septentrionalis Epling (DGO, SIN, SON)

Hyptis sinuata Pohl ex Benth. (CHIS, TAB)

Hyptis spicigera Lam. (CAM, CHIS, COL, GRO, JAL, MÉX, MICH, MOR, NAY, OAX, QROO, VER, YUC)

Hyptis suaveolens (L.) Poit. (CAM, CHIH, CHIS, COL, GRO, JAL, MÉX, MICH, MOR, NAY, OAX, PUE, QRO, QROO, SIN, SLP, SON, TAB, TAM, VER, YUC)

*Hyptis subtilis Epling (COL, GRO, JAL, MÉX, MICH, NAY, OAX, SIN)

*Hyptis tephrodes A. Gray (BCS, NAY, SIN, SON)

* Hyptis tomentosa Poit. (CHIS, GRO, OAX, PUE, VER)

Hyptis urticoides Kunth (BCS, CHIS, COL, DGO, GRO, JAL, MÉX, MICH, NAY, OAX, PUE, SON, VER, ZAC)

Hyptis verticillata Jacq. (CAM, CHIS, HGO, MICH, NAY, OAX, PUE, QRO, QROO, SLP, TAB, TAM, VER, YUC)

Leonotis (1/0/0)

Leonotisnepetifolia (L.) R.Br. (AGS, CAM, CDM, CHIH, CHIS, COL, DGO, GTO, HGO, JAL, MÉX, MICH, MOR, NAY, NL, OAX, PUE, QRO, QROO, SIN, SLP, SON, TAB, TAM, TLX, VER, YUC, ZAC)

\section{Leonorus $(1 / 0 / 0)$}

Leonurus sibiricus L. (CHIS, OAX, PUE, QRO, QROO, TAB, VER, YUC)

\section{Lepechinia (12/6/2)}

Lepechinia cardiophylla Epling (BC)

Lepechinia caulescens (Ortega) Epling (CHIH, CDM, COL, DGO, GRO, GTO, HGO, JAL, MÉX, MICH, NAY, OAX, PUE, QRO, SIN, SLP, SON, TLX, VER, ZAC)

* Lepechinia flammea Mart.Gord. \& Lozada-Pérez (GRO, JAL, OAX)

Lepechinia ganderi Epling (BC)

* Lepechinia glomerata Epling (GRO, JAL)

Lepechinia hastata (A.Gray) Epling (BCS, COL)

* Lepechinia leucophylloides (Ramamoorthy, Hiriart \& Medrano) B.T.Drew, Cacho \& Sytsma (HGO)

Lepechinia mecistandra (Donn.Sm.) H.K.Moon (CHIS, OAX)

* Lepechinia mexicana (S.Schauer) Epling (HGO, OAX, PUE, QRO, SLP)

*Lepechinia nelsonii (Fernald) Epling (DGO, GRO, JAL, MÉX, $\mathrm{MICH}, \mathrm{SIN}$ ) 
Apéndice 1. Continuación.

Lepechinia schiedeana (Schltdl.) Vatke (CHIH, CHIS, DGO, GRO, HGO, JAL, MÉX, NL, OAX, PUE, QRO, TAM, VER)

* Lepechinia yecorana Henrisckson, Fishbein \& T.Van Devender (SON)

\section{Marrubium (1/0/0)}

Marrubium vulgare L. (AGS, BC, CDM, CHIH, COAH, DGO, GTO, HGO, JAL, MÉX, MICH, NL, OAX, PUE, QRO, SLP, SON, TAM, TLX, VER, ZAC)

\section{Marsypianthes(1/0/0)}

Marsypianthes chamaedrys (Vahl) Kuntze (CHIS, GRO, JAL, MICH, NAY, OAX, VER)

\section{Monarda (6/3/1)}

* Monarda bartlettii Standl. (TAM, VER)

Monarda citriodora Cerv. ex Lag. (AGS, CHIH, COAH, DGO, HGO, MICH, NL, SIN, SON, TAM)

*Monarda eplingiana Standl. (COAH)

Monarda fistulosa L. (COAH, HGO, NL, SON, TAM, VER)

*Monarda pringlei Fernald (COAH, NL)

Monarda punctata L. (CHIH, TAM)

\section{Monardella (9/2/1)}

Monardella breweri A.Gray (BC)

Monardella hypoleuca A.Gray (BC)

*Monardella lagunensis M.E.Jones (BC, BCS)

Monardella linoides A.Gray (BC)

Monardella macrantha A.Gray (BC)

Monardella nana A.Gray (BC)

Monardella robisonii Epling ex Munz (BC)

Monardella stoneana Elvin \& A.C.Sanders (BC)

*Monardella thymifolia Greene (BC)

\section{Ocimum (3/0/0)}

Ocimum basilicum L. (CAM, CDM, GRO, JAL, MICH, NL, OAX, PUE, QRO, SON, TAB, VER, YUC)

Ocimum campechianum Mill. (CAM, CHIS, GRO, HGO, MICH, OAX, PUE, QROO, SLP, SON, TAB, TAM, VER, YUC)

Ocimum carnosum (Spreng.) Link \& Otto ex Benth. (CHIS, HGO, OAX, PUE, QRO, SLP, VER)

\section{Physostegia (2/0/0)}

Physostegia correllii (Lundell) Shinners (COAH, NL)

Physostegia virginiana (L.) Benth. (COAH, DGO, SON)

Pogogyne (3/1/1)

Pogogyne nudiuscula A.Gray (BC)

Pogogyne serpylloides (Torr.) A.Gray (BC)

*Pogogyne tenuiflora A.Gray (BC)

Poliomintha (8/5/3)

* Poliomintha bustamanta B.L.Turner (NL)

*Poliomintha conjunctrix Epling \& Wiggins (BC)

*Poliomintha dendritica B.L.Turner $(\mathrm{COAH}, \mathrm{NL})$

Poliomintha glabrescens A.Gray ex Hemsl. (COAH, SLP)

Poliomintha incana (Torr.) A.Gray (CHIH, COAH)

Poliomintha longiflora A.Gray (COAH, NL, TAM, ZAC)

*Poliomintha maderensis Henrisckson $(\mathrm{COAH})$
*Poliomintha marifolia (S.Schauer) A.Gray (GTO, HGO, PUE, SLP)

Prunella (1/0/0)

Prunella vulgaris L. (AGS, CDM, CHIH, CHIS, COAH, DGO, GTO, HGO, JAL, MÉX, MICH, NAY, NL, OAX, PUE, SLP, SON, TLX, VER)

\section{Salvia (306/236/102)}

* Salvia acerifolia B.L.Turner (GRO, JAL, MICH, OAX)

* Salvia adenophora Fernald (OAX)

* Salvia aequidistans Fernald (DGO, JAL, NAY, SIN, SON)

* Salvia agnes Epling (COL, DGO, JAL, MICH, NAY, SIN)

* Salvia alamosana Rose (CHIH, DGO, JAL, NAY, SIN, SON)

* Salvia albicalyx J.G.González (DGO)

Salvia albiflora M.Martens \& Galeotti (CHIS, HGO, OAX, PUE, TAB, VER)

* Salvia albiterrarum J.G.González \& Art.Castro (JAL)

*Salvia albocaerulea Linden (GRO, JAL, MÉX, MICH, MOR)

Salvia altimitrata Epling (CHIS)

* Salvia amarissima Ortega (CDM, CHIS, GTO, HGO, MÉX, MICH, OAX, PUE, QRO, SLP, TLX, VER, ZAC)

*Salvia anastomosans Ramamoorthy (OAX, PUE)

* Salvia angustiarum Epling (JAL, NAY, ZAC)

Salvia apiana Jeps. (BC)

Salvia arizonica A.Gray (CHIH, COAH, SON)

* Salvia arthrocoma Fernald (HGO, PUE, VER)

* Salvia aspera M.Martens \& Galeotti (OAX, PUE)

* Salvia assurgens Kunth (GTO, MÉX, MICH)

* Salvia atropaenulata Epling (GRO, MICH, OAX, VER)

* Salvia axillaris Moc. \& Sessé ex Benth. (AGS, DGO, GTO, HGO, JAL, MÉX, MOR, OAX, PUE, QRO, SLP, VER, ZAC)

Salvia ballotiflora Benth. (AGS, CHIH, COAH, DGO, GTO, HGO, JAL, NL, QRO, SLP, SON, TAM, ZAC)

* Salvia betulifolia Epling (CHIH, DGO, SON)

* Salvia biserrata M.Martens \& Galeotti (VER)

* Salvia blepharophylla Brandegee ex Epling (NL, SLP)

* Salvia boegei Ramamoorthy (PUE)

* Salvia brachyodonta Briq. (AGS, JAL, ZAC)

Salvia brandegeei Munz (BC)

*Salvia breviflora Moc. \& Sessé ex Benth. (GRO, MICH, MOR, OAX, PUE)

* Salvia buchananii Hedge (QRO, SLP)

* Salvia bupleuroides J.Presl ex Benth. (GRO)

Salvia cacaliifolia Benth. (CHIS, OAX)

* Salvia cacomensis J.G.González, J.G.Morales \& J.L.Rodr. (JAL)

* Salvia calderoniae Bedolla \& Zamudio (GTO, QRO)

* Salvia californica Brandegee (BC)

* Salvia candicans M.Martens \& Galeotti (HGO, OAX, PUE, VER)

Salvia carduacea Benth. (BC)

Salvia carnea Kunth (CHIS, GRO, HGO, JAL, MÉX, MICH, MOR, OAX, PUE, VER)

* Salvia carranzae Zamudio \& Bedolla (QRO, SLP) 
Apéndice 1. Continuación.

* Salvia carreyesi J.G.González (JAL)

*Salvia caudata Epling (NL, TAM)

* Salvia cedrosensis Greene (BC, BCS)

* Salvia chalarothyrsa Fernald (COL, JAL, MICH)

*Salvia chamaedryoides Cav. (COAH, HGO, MÉX, MICH, NL, PUE, QRO, SLP, TLX, ZAC)

* Salvia chapalensis Briq. (JAL)

* Salvia chazaorana B.L.Turner (VER)

* Salvia chiapensis Fernald (CHIS)

* Salvia chionopeplica Epling (BC)

* Salvia chionophylla Fernald (COAH)

Salvia cinnabarina M.Martens \& Galeotti (CHIS, COL, GRO, HGO, JAL, MICH, OAX, PUE)

* Salvia clarkcowanii B.L.Turner (GRO, OAX)

Salvia clevelandii (A.Gray) Greene (BC)

* Salvia clinopodioides Kunth (CHIH, DGO, JAL, MÉX, MICH)

*Salvia coahuilensis Fernald (COAH, NL)

Salvia coccinea Buc'hoz ex Etl. (AGS, CAM, CHIH, CHIS, COAH, GTO, HGO, JAL, MICH, NL, OAX, PUE, QRO, QROO, SLP, TAB, TAM, VER, YUC)

* Salvia collinsii Donn.Sm. (CHIS, OAX)

Salvia columbariae Benth. (BC, SON)

* Salvia compacta Kuntze (CHIS, GRO, MÉX, MICH, OAX, PUE)

*Salvia compsostachys Epling (HGO, NL, PUE, QRO, TAM, VER)

*Salvia concolor Lamb. ex Benth. (CDM, CHIS, DGO, GRO, JAL, MÉX, MICH, PUE)

*Salvia confertispicata Fragoso \& Mart.Gord. (GRO, OAX)

*Salvia connivens Epling (GTO, HGO, NL, OAX, PUE, QRO, SLP, TAM)

*Salvia coulteri Fernald (COAH, DGO, NL, SLP)

*Salvia crucis Epling (DGO, JAL, NAY, SIN)

*Salvia cryptodonta Fernald (DGO, JAL, ZAC)

*Salvia cualensis J.G.González (JAL)

Salvia curtiflora Epling (CHIS)

* Salvia curviflora Benth. (GTO, HGO, MÉX, MICH, MOR, PUE, QRO)

* Salvia cyanantha Epling (MICH)

* Salvia darcyi J. Compton (NL)

*Salvia decora Epling (DGO, GRO, JAL, NAY, OAX)

*Salvia dichlamys Epling (GRO, JAL, MÉX, MICH)

*Salvia diegoae Mart.Gord. \& Lozada-Pérez (GRO)

Salvia disjuncta Fernald (CHIS, MÉX, MOR, OAX, PUE)

* Salvia divinorum Epling \& Játiva (OAX)

* Salvia dryophila Epling (MÉX)

* Salvia dugesiana Epling (GTO)

*Salvia dugesii Fernald (GTO)

* Salvia durangensis J.G.González (DGO)

*Salvia durantiflora Epling (GRO)

* Salvia duripes Epling \& Mathias (CHIS)

*Salvia eizi-matudae Ramamoorthy (CHIS)
*Salvia elegans Vahl (AGS, CDM, CHIH, DGO, GRO, GTO, HGO, JAL, MÉX, MICH, MOR, NAY, OAX, PUE, QRO, SIN, SON, TLX, VER, ZAC)

* Salvia emaciata Epling (CHIH, DGO, SON, ZAC)

Salvia eremostachya Jeps. (BC)

* Salvia evadens J.G.González \& Art.Castro (JAL)

* Salvia exilis Epling (GRO)

Salvia farinacea Benth. (COAH, NL, TAM)

Salvia festiva Epling (CHIS)

* Salvia filifolia Ramamoorthy (MÉX)

* Salvia filipes Benth. (HGO)

* Salvia firma Fernald (JAL, NAY)

Salvia flaccida Fernald (CHIS)

* Salvia flaccidifolia Fernald (HGO, PUE)

* Salvia fruticulosa Benth. (OAX, PUE)

*Salvia fulgens Cav. (CDM, GRO, HGO, MÉX, MICH, MOR, PUE, QRO, TLX, VER)

* Salvia fusca Epling (GRO)

* Salvia galloana B.L.Turner (GRO)

*Salvia gesneriiflora Lindl. \& Paxton (AGS, CDM, COL, DGO, HGO, JAL, MÉX, MICH, MOR, PUE, SIN, TLX, ZAC)

*Salvia glabra M.Martens \& Galeotti (HGO, OAX, QRO)

*Salvia glechomifolia Kunth (COAH, GRO, GTO, HGO, MÉX, $\mathrm{MICH}, \mathrm{NL}, \mathrm{OAX}, \mathrm{PUE}, \mathrm{QRO}, \mathrm{SLP}, \mathrm{TAM})$

*Salvia goldmanii Fernald (CHIH, SIN, SON)

* Salvia gonzalezii Fernald (OAX)

* Salvia gravida Epling (MICH)

Salvia greggii A.Gray (CHIH, COAH, DGO, NL, SLP, TAM, ZAC)

* Salvia guadalajarensis Briq. (JAL)

* Salvia guevarae Bedolla \& Zamudio (QRO, SLP)

* Salvia gypsophila B.L.Turner (NL)

*Salvia hamulus Epling (GRO)

*Salvia helianthemifolia Benth. (GRO, GTO, HGO, JAL, MÉX, $\mathrm{MICH}, \mathrm{PUE}, \mathrm{QRO}, \mathrm{SLP}, \mathrm{VER})$

Salvia henryi A.Gray $(\mathrm{CHIH}, \mathrm{NL}, \mathrm{SON})$

* Salvia herbacea Benth. (CHIS, OAX)

* Salvia heterofolia Epling \& Mathias (GRO, MOR)

*Salvia heterotricha Fernald (AGS, DGO, JAL, NAY, ZAC)

* Salvia hidalgensis Miranda (HGO)

* Salvia hintonii Epling (GRO)

*Salvia hirsuta Jacq. (AGS, CDM, DGO, GRO, GTO, HGO, JAL, MÉX, MICH, OAX, QRO, SLP, ZAC)

Salvia hispanica L. (AGS, CHIH, CHIS, COAH, COL, DGO, GRO, GTO, HGO, JAL, MÉX, MICH, MOR, NAY, NL, OAX, PUE, QRO, SON, TAM, VER, ZAC)

Salvia holwayi S.F.Blake (CHIS)

*Salvia ibugana J.G.González (JAL)

* Salvia igualensis Fernald (GRO)

* Salvia indigocephala Ramamoorthy (MICH)

* Salvia infuscata Epling (CHIS, OAX)

*Salvia inornata Epling (PUE) 
Apéndice 1. Continuación.

Salvia involucrata Cav. (GTO, HGO, NL, OAX, PUE, QRO, SLP, TAM, VER)

*Salvia iodantha Fernald (CHIH, COL, DGO, GRO, GTO, HGO, JAL, MÉX, MICH, MOR, NAY, SIN, SON)

* Salvia iodophylla Epling (PUE, VER)

* Salvia ionocalyx Epling (CHIS)

*Salvia jacobi Epling (GRO)

*Salvia jaimehintoniana Ramamoorthy ex B.L.Turner (AGS, DGO, GTO, HGO, NL, QRO, TAM)

Salvia karwinskii Benth. (CHIS, OAX, PUE, VER)

*Salvia keerlii Benth. (GTO, HGO, MICH, NL, OAX, PUE, QRO, SLP, TAM)

*Salvia laevis Benth. (CDM, DGO, GTO, HGO, JAL, MÉX, MICH, MOR, NAY, OAX, PUE, QRO, SLP, TAM, TLX, VER)

*Salvia langlassei Fernald (GRO)

* Salvia languidula Epling (COL, DGO, GRO, JAL, MICH, NAY)

*Salvia lanicalyx Epling (COAH)

*Salvia lapazana B.L.Turner (BCS)

Salvia lasiantha Benth. (CHIS, GRO, HGO, JAL, OAX, PUE, SIN, TAM, VER, ZAC)

Salvia lasiocephala Hook. \& Arn. (CHIH, CHIS, COL, DGO, GRO, JAL, MÉX, MICH, NAY, OAX, PUE, SIN, SON, VER)

Salvia lavanduloides Kunth (AGS, CDM, CHIH, CHIS, COAH, COL, DGO, GRO, GTO, JAL, MÉX, MICH, MOR, NAY, OAX, PUE, SIN, VER, ZAC)

*Salvia leninae Epling (GRO)

*Salvia leptostachys Benth. (AGS, CDM, DGO, GRO, GTO, JAL, MÉX, MICH, MOR, NAY, OAX, PUE, ZAC)

Salvia leucantha Cav. (CHIS, COAH, COL, GRO, GTO, JAL, MÉX, MICH, MOR, OAX, PUE, QRO, SLP, TLX, VER, ZAC)

Salvia leucochlamys Epling (CHIS)

Salvia leucophylla Greene (BC)

*Salvia lineata Benth. (OAX, PUE)

* Salvia littae Vis. (OAX)

*Salvia longispicata M.Martens \& Galeotti (CHIH, CHIS, COL, DGO, GRO, GTO, HGO, JAL, MÉX, MICH, MOR, NAY, NL, OAX, PUE, SIN, SLP, VER)

*Salvia longistyla Benth. (DGO, GRO, GTO, JAL, MÉX, MICH, MOR, NL, OAX, PUE, VER)

*Salvia lophanthoides Fernald (GRO, OAX)

*Salvia lozani Fernald (HGO)

Salvia lycioides A.Gray (CHIH, COAH, DGO, GTO, NL, QRO, SLP, ZAC)

*Salvia macellaria Epling (AGS, COAH, DGO, JAL, NL, SLP, ZAC)

*Salvia madrensis Seem. (DGO, SIN)

*Salvia madrigalii Zamudio \& Bedolla $(\mathrm{MICH})$

* Salvia malvifolia Epling \& Játiva (BCS)

* Salvia manantlanensis Ramamoorthy (JAL)

Salvia mazatlanensis Fernald (SIN)

* Salvia mcvaughii Bedolla, Lara Cabrera \& Zamudio (GRO, MOR)
*Salvia meera Ramamoorthy ex J.G.González \& Santana Mich. (JAL)

* Salvia melissodora Lag. (AGS, CDM, CHIH, DGO, GRO, GTO, HGO, JAL, MÉX, MICH, NAY, OAX, PUE, QRO, TAM, VER, ZAC)

Salvia mellifera Greene (BC)

* Salvia mexiae Epling (JAL)

* Salvia mexicana L. (AGS, CDM, CHIH, CHIS, COL, DGO, GRO, GTO, HGO, JAL, MÉX, MICH, MOR, NAY, OAX, PUE, QRO, SIN, SLP, SON, TAM, VER, ZAC)

Salvia microphylla Kunth (AGS, CDM, CHIH, CHIS, COAH, COL, DGO, GRO, GTO, HGO, JAL, MÉX, MICH, NAY, NL, OAX, PUE, QRO, SLP, SON, TAM, TLX, VER, ZAC)

Salvia miniata Fernald (CHIS)

Salvia misella Kunth (AGS, BCS, CAM, CHIH, CHIS, COL, DGO, GRO, GTO, HGO, JAL, MÉX, MICH, MOR, NAY, NL, OAX, PUE, QRO, QROO, SIN, SON, TAM, VER, YUC, ZAC)

Salvia mocinoi Benth. (CDM, CHIS, GRO, JAL, MÉX, MICH, MOR, NAY, OAX, PUE, VER)

* Salvia modica Epling (DGO)

Salvia mohavensis Greene (SON)

* Salvia monantha Brandegee ex Epling (DGO, SIN, SON)

* Salvia monclovensis Fernald (COAH, NL)

* Salvia moranii B.L.Turner (BCS)

* Salvia muelleri Epling (COAH, NL)

Salvia munzii Epling (BC, SON)

* Salvia muscarioides Fernald (CHIH, MOR, SON)

Salvia nana Kunth (AGS, DGO, GTO, JAL, MÉX, OAX, PUE, SLP, VER, ZAC)

* Salvia nervata M.Martens \& Galeotti (CHIS, OAX)

* Salvia nitida (M.Martens \& Galeotti) Benth. (GRO, OAX)

* Salvia oaxacana Fernald (OAX, PUE, VER)

Salvia oblongifolia M.Martens \& Galeotti (CHIS, OAX)

Salvia occidentalis Sw. (CAM, CHIS, GRO, JAL, MICH, NAY, OAX, PUE, QROO, SIN, TAB, TAM, VER, YUC)

* Salvia occidua Epling (OAX, PUE)

* Salvia odam J.G.González (DGO)

* Salvia omissa J.G.González (JAL)

*Salvia oreopola Fernald (CDM, GRO, MÉX, MOR, OAX, PUE)

* Salvia oresbia Fernald (NL, SLP)

Salvia pachyphylla Epling ex Munz (BC)

* Salvia palealis Epling (GRO)

*Salvia palmeri A.Gray (CHIH, DGO, SIN, SON)

* Salvia pannosa Fernald (OAX, PUE)

Salvia pansamalensis Donn.Sm. (CHIS)

Salvia parryi A.Gray (SON)

* Salvia patens Cav. (AGS, CHIS, GRO, GTO, HGO, JAL, MÉX, MICH, PUE, QRO, SLP, VER)

* Salvia paupercula Epling (PUE)

* Salvia peninsularis Brandegee (BCS)

* Salvia pennellii Epling (NL, SLP, TAM)

*Salvia perblanda Epling (GRO) 
Apéndice 1. Continuación.

*Salvia pericona B.L.Turner (OAX)

* Salvia perlonga Fernald (GRO)

* Salvia pexa Epling (OAX, PUE)

* Salvia pineticola Epling (VER)

* Salvia platycheila A.Gray (BCS)

*Salvia platyphylla Briq. (JAL, NAY, ZAC)

* Salvia plurispicata Epling (MICH)

*Salvia podadena Briq. (AGS, COAH, DGO, GRO, HGO, NL, OAX, PUE, QRO, SLP, ZAC)

Salvia polystachya Cav. (AGS, CDM, CHIS, COL, DGO, GRO, GTO, HGO, JAL, MÉX, MICH, MOR, NAY, OAX, PUE, QRO, SIN, TLX, VER, ZAC)

*Salvia potus Epling (COAH, NL)

* Salvia praestans Epling (GRO)

* Salvia prasiifolia Benth. (NAY)

*Salvia pringlei B.L.Rob. \& Greenm. (JAL, NAY, SIN)

*Salvia protracta Benth. (CHIS, GRO, MICH, MOR, OAX, VER)

* Salvia pruinosa Fernald (DGO, JAL)

*Salvia prunelloides Kunth (AGS, CDM, CHIH, CHIS, COAH, DGO, GRO, GTO, HGO, JAL, MÉX, MICH, MOR, NAY, NL, OAX, PUE, QRO, SIN, SLP, SON, TAM, TLX, VER, ZAC)

*Salvia prunifolia Fernald (DGO, JAL, NAY, ZAC)

* Salvia pseudopallida Epling (COAH)

* Salvia pugana J.G.González \& Art.Castro (JAL)

*Salvia pulchella DC. (CDM, GTO, JAL, MÉX, MICH)

*Salvia punicans Epling (GRO, OAX)

*Salvia purepecha Bedolla, Lara Cabrera \& Zamudio (JAL, $\mathrm{MICH})$

Salvia purpurea Cav. (AGS, CHIS, COL, DGO, GRO, GTO, HGO, JAL, MÉX, MICH, MOR, NAY, OAX, PUE, SIN, VER, ZAC)

* Salvia purpusii Brandegee (COAH, DGO)

* Salvia pusilla Fernald (OAX, PUE)

*Salvia quercetorum Epling (JAL)

*Salvia ramamoorthyana Espejo (CDM, GRO, JAL, MÉX, MICH, MOR, OAX)

* Salvia ramirezii J.G.González (JAL)

*Salvia raveniana Ramamoorthy (GRO, OAX)

Salvia recurva Benth. (CHIS, GRO, HGO, OAX, PUE, VER)

Salvia reflexa Hornem. (AGS, CHIH, COAH, DGO, GTO, HGO, JAL, MÉX, MICH, NL, OAX, PUE, QRO, SLP, SON, TAM, ZAC)

Salvia regla Cav. (AGS, CHIH, COAH, DGO, GTO, HGO, JAL, MÉX, MICH, NL, OAX, PUE, QRO, SLP, SON, TAM, VER, ZAC)

Salvia reptans Jacq. (AGS, CDM, CHIH, CHIS, COAH, COL, GRO, GTO, HGO, JAL, MÉX, MICH, MOR, NAY, NL, OAX, PUE, QRO, SLP, ZAC)

* Salvia rhyacophila (Fernald) Epling (CHIS, GRO, MÉX, MICH, MOR, OAX, PUE)

*Salvia richardsonii B.L.Turner (TAM)

* Salvia robertoana Mart.Gord. \& Fragoso (OAX)
Salvia roemeriana Scheele $(\mathrm{CHIH}, \mathrm{COAH}, \mathrm{NL}, \mathrm{TAM})$

* Salvia rogersiana Ramamoorthy ex J.G.González \& Cuevas (JAL)

*Salvia roscida Fernald (COL, DGO, GRO, JAL, MICH, MOR, NAY, OAX, SIN, SON)

* Salvia rosei Fernald (JAL, NAY, ZAC)

* Salvia rostellata Epling (GRO, JAL, NAY)

Salvia rubiginosa Benth. (CHIS, OAX, PUE, VER)

* Salvia rubropunctata B.L.Rob. \& Fernald (SON)

* Salvia rzedowskii Ramamoorthy (MÉX)

* Salvia sanctae-luciae Seem. (NAY, SIN)

* Salvia santanae Ramamoorthy ex J.G.González \& Guzm.-Hern. $(\mathrm{COL}, \mathrm{JAL})$

* Salvia scaposa Epling (GRO, MÉX)

* Salvia seemannii Fernald (CHIH, DGO, SIN, SON)

* Salvia semiatrata Zucc. (OAX)

* Salvia semiscaposa Epling ex Fragoso \& Mart.Gord. (MÉX)

Salvia serotina L. (CAM, CHIS, QROO, TAB, YUC)

* Salvia serpyllifolia Fernald (JAL, SLP, ZAC)

*Salvia sessei Benth. (COL, GRO, HGO, JAL, MÉX, MICH, MOR, OAX, PUE, QRO, TAM)

* Salvia setulosa Fernald (JAL, MÉX, MICH, MOR, OAX, PUE, VER)

* Salvia schaffneri Fernald (SLP)

Salvia shannonii Donn.Sm. (CHIS, VER)

* Salvia sharpii Epling \& Mathias (NL, SLP, TAM)

* Salvia similis Brandegee (BCS, SON)

Salvia simulans Fernald (HGO, PUE)

* Salvia sinaloensis Fernald (NAY, SIN)

* Salvia sphacelifolia Epling (SIN)

* Salvia stachyoides Kunth (CDM, MICH, PUE, QRO, TLX, VER)

* Salvia stolonifera Benth. (GRO, OAX)

*Salvia subhastata Epling (GRO, MICH)

Salvia subincisa Benth. (AGS, CHIH, DGO, SON, TAM, ZAC)

* Salvia subpatens Epling (GRO, JAL, MÉX, MICH)

Salvia subrubens Epling (CHIS)

Salvia summa A.Nelson $(\mathrm{CHIH})$

* Salvia synodonta Epling (MICH)

* Salvia tehuacana Fernald (OAX, PUE)

* Salvia tenorioi Ramamoorthy ex B.L.Turner (PUE)

* Salvia teresae Fernald (JAL, MÉX, NAY)

* Salvia tetramerioides Mart.Gord., Fragoso \& García-Peña (OAX)

Salvia texana (Scheele) Torr. (CHIH, COAH, NL)

* Salvia textitlana B.L.Turner (OAX)

* Salvia thymoides Benth. (OAX, PUE, VER)

*Salvia thyrsiflora Benth. (COL, GRO, JAL, MICH, NAY)

*Salvia tilantongensis J.G.González \& Aguilar-Sant. (OAX)

Salvia tiliifolia Vahl (AGS, CDM, CHIH, CHIS, COAH, COL, DGO, GRO, GTO, HGO, JAL, MÉX, MICH, MOR, NAY, NL, OAX, PUE, QRO, SIN, SLP, TAM, VER, ZAC)

* Salvia tonalensis Brandegee (CHIS) 
Apéndice 1. Continuación.

* Salvia tonaticensis Ramamoorthy ex Lara Cabrera, Bedolla \& Zamudio (GRO, MÉX)

* Salvia topiensis J.G.González (DGO)

* Salvia trichostephana Epling (SIN)

*Salvia tricuspidata M.Martens \& Galeotti (OAX)

Salvia tubifera Cav. (CHIS, DGO, GRO, HGO, JAL, MÉX, OAX, PUE, VER)

*Salvia turneri Ramamoorthy (GRO)

* Salvia tuxtlensis Ramamoorthy (VER)

*Salvia umbraticola Epling (PUE)

* Salvia umbratilis Fernald (GRO)

*Salvia unicostata Fernald (JAL, NL, SLP, TAM, ZAC)

*Salvia univerticillata Ramamoorthy ex Klitg. (CHIS)

Salvia urica Epling (CHIS, GRO, HGO, OAX)

* Salvia urolepis Fernald (NL, QRO, TAM)

* Salvia uruapana Epling (COL, GRO, JAL, MÉX, MICH)

Salvia vaseyi (Porter) Parish (BC)

*Salvia vazquezii Iltis \& Ramamoorthy $(\mathrm{COL}, \mathrm{JAL}, \mathrm{MICH})$

* Salvia venturana B.L.Turner (PUE)

*Salvia verecunda Epling ex M.E.Jones (CHIH, DGO, SON)

*Salvia veronicifolia A.Gray (JAL, NAY, ZAC)

* Salvia villosa Fernald (NL, SLP)

Salvia vitifolia Benth. (CHIS, GRO, OAX, PUE, VER)

Salvia wagneriana Pol. (CHIS)

Salvia whitefoordiae Klitg. (CHIS)

Salvia whitehousei Alziar (CHIS)

* Salvia wixarika J.G.González (JAL)

*Salvia xalapensis Benth. (CHIS, HGO, OAX, PUE, VER)

*Salvia xolocotzii Bedolla \& Zamudio (QRO)

* Salvia zamoranensis Zamudio \& Bedolla (GTO, QRO)

* Salvia zaragozana B.L.Turner (NL)

Scutellaria (61/40/24)

* Scutellaria alta M.E.Jones $(\mathrm{CHIH})$

* Scutellaria anomala Epling (NL)

* Scutellaria aramberrana B.L.Turner (NL)

* Scutellaria aurea B.L.Rob. \& Greenm. (OAX)

* Scutellaria bartlettii B.L.Turner (NL, TAM)

* Scutellaria blepharophylla Epling (GRO, JAL, MÉX, MICH)

* Scutellaria carmenensis Henrisckson $(\mathrm{COAH})$

Scutellaria chalicophila Loes. (CHIS)

* Scutellaria cuevasiana J.G.González \& A.Vázquez (JAL)

* Scutellaria cylindriflora Epling \& Játiva (SIN)

* Scutellaria chiangii B.L.Turner (COAH)

Scutellaria drummondii Benth. (COAH, NL, TAM)

Scutellaria dumetorum Schltdl. (CDM, CHIS, COL, GRO, GTO, HGO, JAL, MÉX, MICH, MOR, NL, OAX, PUE, QRO, SLP, VER)

Scutellaria formosa Leonard (GRO, OAX, VER)

* Scutellaria fraxinea Epling (NL, TAM)

* Scutellaria fruticetorum Epling (NL, SLP, TAM)

Scutellaria gaumeri Leonard (CAM, QROO, SLP, VER, YUC)

scutellaria guatemalensis Leonard (CHIS)
Scutellaria havanensis Jacq. (TAM, VER)

* Scutellaria hintoniana Epling (MÉX, OAX)

* Scutellaria hintoniorum Henrisckson (NL)

* Scutellaria hispidula B.L.Rob. (CHIH, COAH, DGO, JAL, MÉX, $\mathrm{MICH}, \mathrm{NL}, \mathrm{PUE}, \mathrm{SLP}, \mathrm{SON}, \mathrm{ZAC})$

* Scutellaria horridula Epling (SON)

* Scutellaria jaliscana Epling (JAL, MICH)

Scutellaria longifolia Benth. (CHIS, GRO)

Scutellaria lundellii Epling (CHIS)

* Scutellaria lutilabia T.M.Lane \& G.L.Nesom (NL)

* Scutellaria macra Epling (GTO, HGO, PUE, SLP, VER)

Scutellaria mexicana (Torr.) A.J.Paton (BC, BCS, CHIH, COAH, DGO)

Scutellaria microphylla Moc. \& Sessé ex Benth. (COAH, NL, TAM)

Scutellaria mociniana Benth. (CHIS, VER)

* Scutellaria modesta Játiva \& Epling (SLP)

* Scutellaria molanguitensis Hiriart (HGO)

* Scutellaria monterreyana B.L.Turner (NL)

* Scutellaria multiflora Benth. (GRO, MÉX, MICH)

*Scutellaria mulleri B.L.Turner (COAH)

* Scutellaria muzquiziana B.L.Turner (COAH)

* Scutellaria oaxacana Greenm. (CHIS, OAX, PUE, VER)

Scutellaria orichalcea Donn.Sm. (CHIS, OAX, TAB, VER)

Scutellaria orizabensis Epling (CHIS, PUE, VER)

Scutellaria ovata Hill (NL)

*Scutellaria pallidiflora Epling (JAL)

* Scutellaria petersoniae B.L.Turner \& Reveal (GRO)

* Scutellaria potosina Brandegee (AGS, CHIH, COAH, GTO,

HGO, MICH, NL, PUE, QRO, SLP, SON, TAM, ZAC)

* Scutellaria pseudocoerulea Briq. (HGO, PUE, QRO, SLP, VER)

Scutellaria racemosa Pers. (CHIS, JAL, MICH, SLP, VER)

* Scutellaria rosei Fernald (NAY)

* Scutellaria russelioides Epling (DGO, SIN)

Scutellaria seleriana Loes. (CAM, CHIS, NL, OAX, PUE, QRO,

QROO, SLP, TAB, TAM, VER, YUC)

* Scutellaria serboana B.L.Turner (OAX)

* Scutellaria sipilensis Cuevas (JAL)

* Scutellaria splendens Link, Klotzsch \& Otto (CHIS, OAX, TAB, VER)

* Scutellaria stachyoides Epling (SON)

* Scutellaria stewartii B.L.Turner (COAH)

* Scutellaria sublitoralis J.G.González (JAL, NAY)

* Scutellaria suffrutescens S.Watson (COAH, NL, TAM)

Scutellaria texana B.L.Turner $(\mathrm{COAH}, \mathrm{NL})$

Scutellaria tuberosa Benth. (BC)

Scutellaria vitifolia Brandegee (CHIS)

* Scutellaria wendtii Henrisckson (COAH)

Scutellaria wrightii A.Gray $(\mathrm{COAH})$

Stachys (45/33/14)

Stachys agraria Schltdl. \& Cham. (AGS, CDM, CHIH, CHIS, 
Apéndice 1. Continuación.

COAH, COL, DGO, GRO, GTO, HGO, JAL, MÉX, MICH, MOR, NAY, NL, OAX, PUE, QRO, SIN, SLP, SON, TAM, VER)

Stachys ajugoides Benth. (BC)

* Stachys al botomentosa Ramamoorthy (GTO, HGO, SLP)

* Stachys aristata Greenm. (JAL)

* Stachys arriagana B.L.Turner (SLP, ZAC)

* Stachys biflora Hook. \& Arn. (AGS, CHIH, COAH, GRO, HGO, JAL, MÉX, MICH, NAY, NL, PUE, QRO, SIN, SLP, TAM, VER)

Stachys bigelovii A.Gray (AGS, BC, CHIH, COAH, DGO, NAY, NL, SLP, SON, ZAC)

*Stachys boraginoides Schltdl. \& Cham. (CHIS, GRO, HGO, $\mathrm{MICH}, \mathrm{OAX}, \mathrm{PUE}, \mathrm{QRO}, \mathrm{VER})$

Stachys coccinea Ortega (AGS, BCS, CDM, CHIH, CHIS, COAH, COL, DGO, GRO, GTO, HGO, JAL, MÉX, MICH, MOR, NAY, OAX, PUE, QRO, SIN, SLP, SON, VER, ZAC)

* Stachys collina Brandegee (PUE)

Stachys costaricensis Briq. (CHIS, VER)

Stachys drummondii Benth. (NL, TAM)

Stachys eriantha Benth. (CDM, HGO, MÉX, MICH, MOR, PUE, QRO, SLP, TLX, VER)

* Stachys exilis Epling (OAX)

* Stachys globosa Epling (MÉX, QRO, SLP)

* Stachys harkerae J.G.González (JAL, ZAC)

* Stachys harleyana A.Pool (CHIS)

* Stachys herrerana Rzed. \& Calderón (HGO)

* Stachys hintoniorum B.L.Turner (COAH, NL)

* Stachys inclusa Epling (OAX, PUE)

* Stachys keerlii Benth. (GTO, MÉX, MICH, OAX, PUE, VER)

* Stachys langmaniae Rzed. \& Calderón (NL, TAM)

Stachys lindenii Benth. (CHIS, GRO, MICH, OAX, PUE, QRO, TAB, VER)

*Stachys manantlanensis B.L.Turner (JAL, MICH)

* Stachys mohinora B.L.Turner (CHIH)

* Stachys moorei B.L.Turner (HGO)

*Stachys nepetifolia Cav. (CDM, GTO, HGO, MÉX, QRO, SLP, VER)

Stachys nubilorum Epling (CHIS)

* Stachys pacifica B.L.Turner (COL, JAL, MICH, NAY, SIN, SON)

* Stachys parvifolia M.Martens \& Galeotti $(\mathrm{CHIH}, \mathrm{CHIS}, \mathrm{COAH}$, GTO, HGO, MÉX, MICH, QRO, TAM, VER)

* Stachys penanevada B.L.Turner (NL, TAM)

Stachys pilosissima M.Martens \& Galeotti (CHIS, COL, JAL, MÉX, MICH, OAX, PUE, VER)

* Stachys pringlei Greenm. (HGO, PUE, QRO, VER)

Stachys radicans Epling (CDM, CHIS, MÉX, MICH, VER)

* Stachys rotundifolia Moc. \& Sessé ex Benth. (CDM, CHIS, MÉX, MICH, MOR, OAX, PUE, VER)
*Stachys sanchezii Rzed. \& A.García (CDM, MÉX, MICH, PUE)

* Stachys sandersii B.L.Turner (SLP)

Stachys stebbinsii G.A.Mulligan \& D.B.Munro (BC)

* Stachys tamaulipana B.L.Turner (NL, TAM)

* Stachys tenerrima Epling (BCS)

* Stachys tlaxiacana B.L.Turner (OAX)

* Stachys torresii B.L.Turner (OAX)

* Stachys turneri Rzed. \& Calderón (GTO)

* Stachys venulosa Greene (DGO)

* Stachys vulnerabilis Rzed. \& Calderón (NL)

Tetraclea (1/0/0)

Tetraclea coulteri A.Gray (AGS, CHIH, COAH, DGO, GTO, NL, SLP, SON, TAM, ZAC)

\section{Teucrium (6/2/1)}

Teucrium canadense L. (CAM, CHIH, COAH, GTO, NL)

* Teucrium coahuilanum B.L.Turner (COAH, NL)

Teucrium cubense Jacq. (AGS, BC, CAM, CHIH, COAH, DGO, GTO, JAL, NL, PUE, QRO, QROO, SIN, SLP, SON, TAB,

TAM, VER, YUC, ZAC)

Teucrium glandulosum Kellogg (BC, BCS, SIN, SON)

* Teucrium townsendii Vasey \& Rose (COL)

Teucrium vesicarium Mill. (CAM, CHIS, NL, OAX, PUE, QROO, SIN, SLP, TAB, VER, YUC)

Trichostema $(8 / 2 / 1)$

Trichostema arizonicum A.Gray $(\mathrm{CHIH}, \mathrm{SON})$

Trichostema austromontanum F.H.Lewis (BC)

Trichostema lanatum Benth. (BC)

Trichostema lanceolatum Benth. (BC)

* Trichostema mexicanum Epling (COAH)

Trichostema micranthum A.Gray (BC)

Trichostema parishii Vasey (BC)

* Trichostema purpusii Brandegee (OAX, PUE)

Vitex (4/2/0)

Vitex gaumeri Greenm. (CAM, CHIS, GRO, MOR, QROO, TAB, YUC)

Vitex hemsleyi Briq. (CHIS, COL, GRO, JAL, MICH, NAY, OAX)

*Vitex mollis Kunth (BC, BCS, CHIH, COL, DGO, GRO, JAL, MÉX, MICH, MOR, NAY, OAX, PUE, SIN, SON,VER, YUC, ZAC)

*Vitex pyramidata B.L.Rob. (DGO, GRO, JAL, MÉX, MICH, MOR, NAY, PUE, SIN, SON)

Volkameria (2/0/0)

Volkameria aculeata L. (QROO)

Volkameria ligustrina Jacq. (CAM, CHIS, OAX, PUE, QROO, TAB, TAM, VER, YUC)

Warnockia (1/0/0)

Warnockia scutellarioides (Engelm. \& A.Gray) M.W.Turner $(\mathrm{COAH})$ 